\title{
Die Absorption. Anhäufung von Fluorcalcium, Kalk, Phosphaten in fossilen Knochen.
}

\author{
Von \\ J. M. van Bemmelen. \\ Unter Mitwirkung von Dr. E. A. KцовBIE. \\ Mit 4 Figuren im Text.
}

\section{Einleitung.}

Die nie ruhende chemische Metamorphose in der Erdkruste kann einerseits von dem Gesichtspunkte einer fortwährenden Anhäufung, andererseits dem einer fortwährenden Zerstreuung der chemischen Elemente oder ihrer Verbindungen betrachtet werden.

Die Zerstreuung kommt durch Verwitterung, Auflösung u. s. w. zu stande. Die Anhänfung entsteht an bestimmten Stellen durch Absetzung und Unlöslichwerden der vom Wasser mitgeführten oder gelösten Bestandteile. Die Ursachen davon sind verschiedene.

Es kommt mir vor, dafs die Erscheinungen der Zerstreuung bis jetzt, wenn auch noch sehr unvollkommen, doch etwas mehr untersucht worden sind als die der Anhäufung.

Wenn sich aus einer Lösung oder einem flüssigen Komplex gewisse Bestandteile absetzen, dann sind Zusmmensetzung des Gemisches, Konzentration ( $=$ Gehalt) jedes Bestandteiles, Temperatur und äufserer Druck die wirkenden Faktoren. Mit einem eingehenderen Studium dieser Faktoren hat man in den letzten Jahren einen Anfang gemacht. Wenn jedoch die Anhäufung eines gewissen Bestandteiles darauf beruht, dals dieser sich aus der Lösung auf gewisse Substanzen in einer Erdschicht absetzt, oder davon chemisch gebunden wird, dann können wir unterscheiden: 
1. den Fall, dafs eine Austauschung stattfindet zwischen einem oder mehreren Bestandteilen der Schichtsubstanz und denjenigen des Erdwassers (die gewöhnliche chemische Metamorphose); 2. die Absetzung von bestimmten Bestandteilen aus der Lösung an eine Substanz der Erdschicht - sei es, dals diese damit eine komplexe chemische Verbindung, eine Absorptionsverbindung, oder nur ein mechanisches Anhaften bildet. Es kommen dabei wahrscheinlich besondere Anziehungen ins Spiel, die eine Anhäufung eines gewissen chemischen Elementes oder einer chemischen Verbindung desselben zur Folge haben. Diese Erscheinungen wurden wohl am wenigsten erforscht, und es scheint erwünscht, neues Beobachtungsmaterial zu sammeln.

$\mathrm{Zu}$ diesen Erscheinungen gehört z. B. die Anhäufung von Fluor (als $\mathrm{CaF}_{2}$ ) in phosphorsaurem Kalk. Nun hatte ich Gelegenheit nicht allein die Anhäufung von Fluor, sondern auch von Kalk, kohlensaurem und phosphorsaurem Kalk in einem fossilen Knochen $z \mathfrak{u}$ beobachten.

\section{Analyse.}

Die mikroskopische Untersuchung von Quer- und Längsschliffen unter Anwendung von verdünnter Salzsäure ergab, dafs die Lamellen (HAFER'schen, Zwischen- und Grund-), die Beinhöhlen in den Lamellen (früher Beinkörperchen genannt) und die HaFER'schen Kanäle (also die ganze Struktur) noch vollständig sichtbar waren, namentlich wenn der Schliff mit Alkohol befeuchtet, oder mit etwas verdünnter Salzsäure kurz behandelt war (Fig. 1-4).

An der Aufsenwand des Knochens befindet sich eine damit verwachsene harte Kruste von $\mathrm{CaCO}_{3}$. Im kompakten Knochenteil sind die HATER'schen Kanäle mit weifsem, krystallinischem, kohlensaurem Kalk gefüllt. Bei Befeuchtung mit verdünnter Salzsäure entwichen die $\mathrm{CO}_{2}$-Blasen am meisten aus diesen Kanälchen und wurden dieselben leer. Die äufsere Schicht hat eine dunkelbraune Farbe; viele Kanäle in derselben sind mit Pyrit ausgefüllt (Fig. 1 und 2), so dals der Pyrit das Vorkommen einer dendritischen Bildung hat. An einzelnen Stellen hat sich die Pyritbildung bis ins Gewebe ausgebreitet.

Die innere Schicht des kompakten Teiles ist hellbraungelb gefärbt und ist dadurch von der äufseren Schicht unterschieden; sie ist frei oder fast frei von Pyrit.

Beim Übergảng des kompakten Teiles in den spongiösen tritt der Pyrit stellenweise wieder auf. Die Höhlen sind ganz mit Kalk- 
Fig. 1.

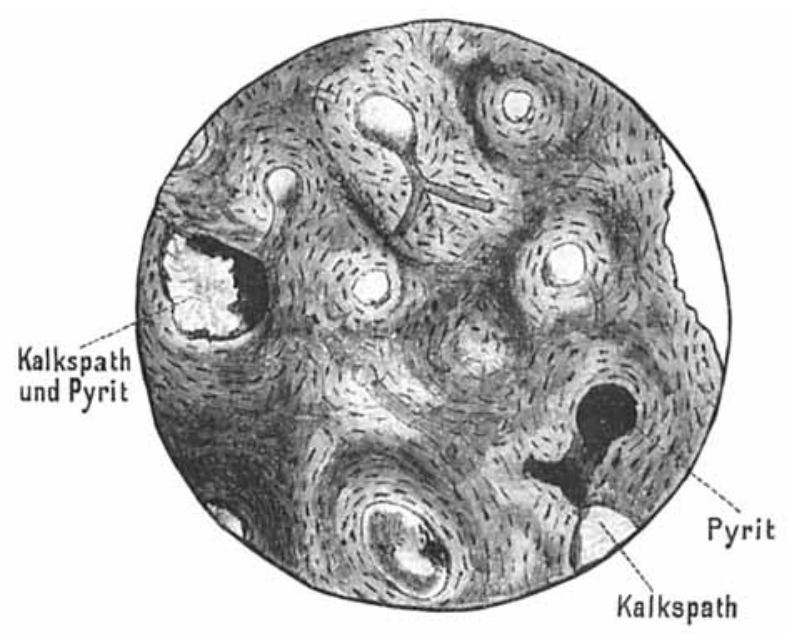

Querschnitt. Kompakter Teil. Äufsere Schicht.

Fig. 2.

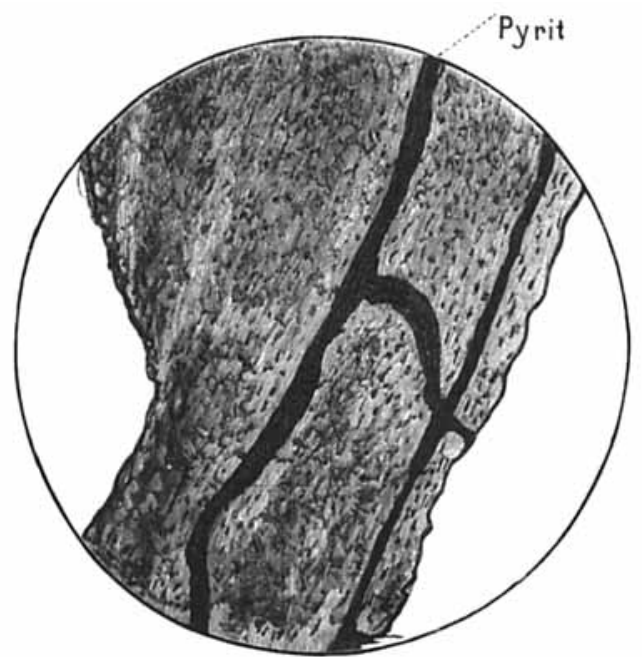

Längsschnitt. Kompakter Teil. Äufsere Schicht. 
Fig. 3.

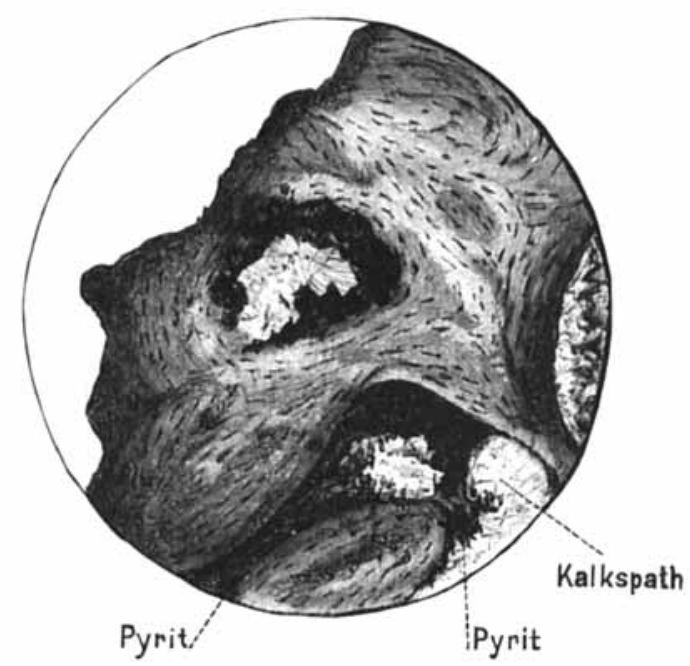

Querschnitt. Übergang vom kompakten in den spongiösen Teil.

Fig. 4.

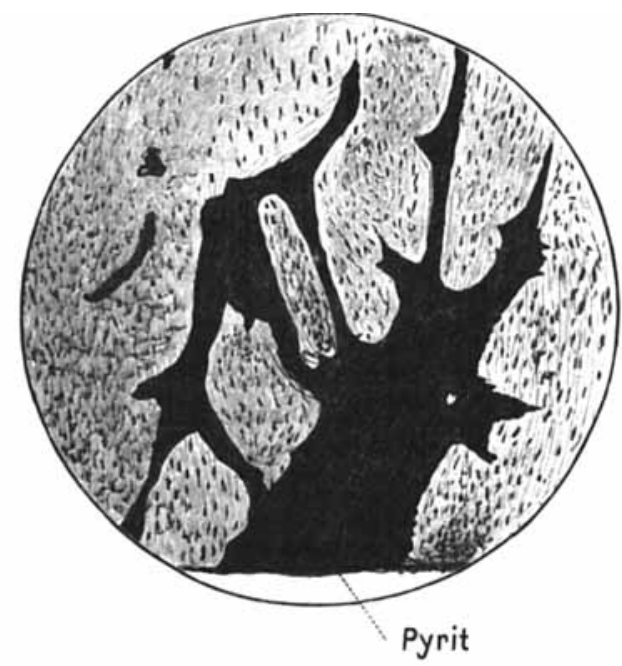

Längsschnitt. Übergang vom kompakten in den spongiösen Teil. 
spat und oft auch mit Pyrit ausgefüllt (Fig. 3 und 4). In der Axialhöhle des spongiösen Knochenteils ist das Gewebe ganz oder teilweise verschwunden, und die dadurch entstandene Höhle ganz oder teilweise mit gröfseren Krystallen von $\mathrm{CaCO}_{3}$ bekleidet, die wieder einige Pyriteinschlüsse enthalten.

Die Versuchssubstanz $(A)$ bestand aus der gelbbraunen kompakten Knochensubstanz, möglichst frei von dem spongiösen Teil und den darin vorhandenen Absetzungen. Die Versuchssubstanz $(B)$ war nicht frei davon, womit die Ergebnisse der Analyse in Einklang sind; denn $B$ ergiebt viel mehr kohlensauren Kalk und Pyrit als $A$. Eine dritte Portion $C$ enthielt wenig Pyrit, aber etwas mehr $\mathrm{CaCO}_{3}$ wie $A$. Eine vierte Portion $D$ war aus der äufseren dunkelbraunen Schicht genommen. Stoff $A$ und Stoff $B$ wurden mit Wasser, mit nachfolgenden kleinen Mengen verdünnter Essigsäure, bis sich nichts mehr löste, mit verdünnter Salzsäure, mit Salpetersäure ausgezogen, und diese Auszüge qualitativ untersucht. Eine möglichst genaue quantitative Analyse war unentbehrlich, um zu untersuchen, ob neben Fluorcalcium noch andere Bestandteile in der Knochensubstanz aufgenommen waren, und ob diese von den eingelagerten Substanzen $\left(\mathrm{CaCO}_{3}\right.$, Pyrit) zu unterscheiden seien. Ich erlaube mir darum auch die befolgten Methoden und erhaltenen Zahlen etwas ausführlicher mitzuteilen, damit sich die erreichte Genauigkeit daraus beurteilen lälst (siehe Anhang).

Die folgende Tabelle enthält die Zahlen der quantitativen Analyse. Neben den Prozentzahlen sind die Äquivalentzahlen gegeben, wie ich das seit vielen Jahren gethan habe. ${ }^{1}$

1 Siehe „Bodenuntersuchungen in den Niederlanden" (Landwirtschaftliche Versuchsstutionen [1866] 8, 278 u. 279). Bei der Analyse von Lösungen und Gemischen, wie natürlichen Wässern, organischen Flüssigkeiten, Ackererden, Mineralien, die isomorphe oder amorphe Gemische darstellen u. s. w., - im all: gemeinen bei Gemischen, die sich mechanisch nicht trennen lassen - scheint es mir unbedingt nötig, neben den Prozentzahlen die Äquivalentzahlen zu schreiben. Die Prozentzahlen haben eigentlich keinen Wert, sie bekommen diesen erst durch die Äquivalentzahlen und durch die Ergebnisse der qualitativen und der mikroskopischen Analyse; denn auch die Berechnungen von bestimmten Salzen aus diesen Äquivalentzahlen sind immer willkürlich, wenn sie eine wässerige oder andere Lösung betreffen. Für feste Gemische können sie nur Wert haben, insofern die Untersuchung Beweise für die Anwesenheit bestimmter Salze ergieht. 


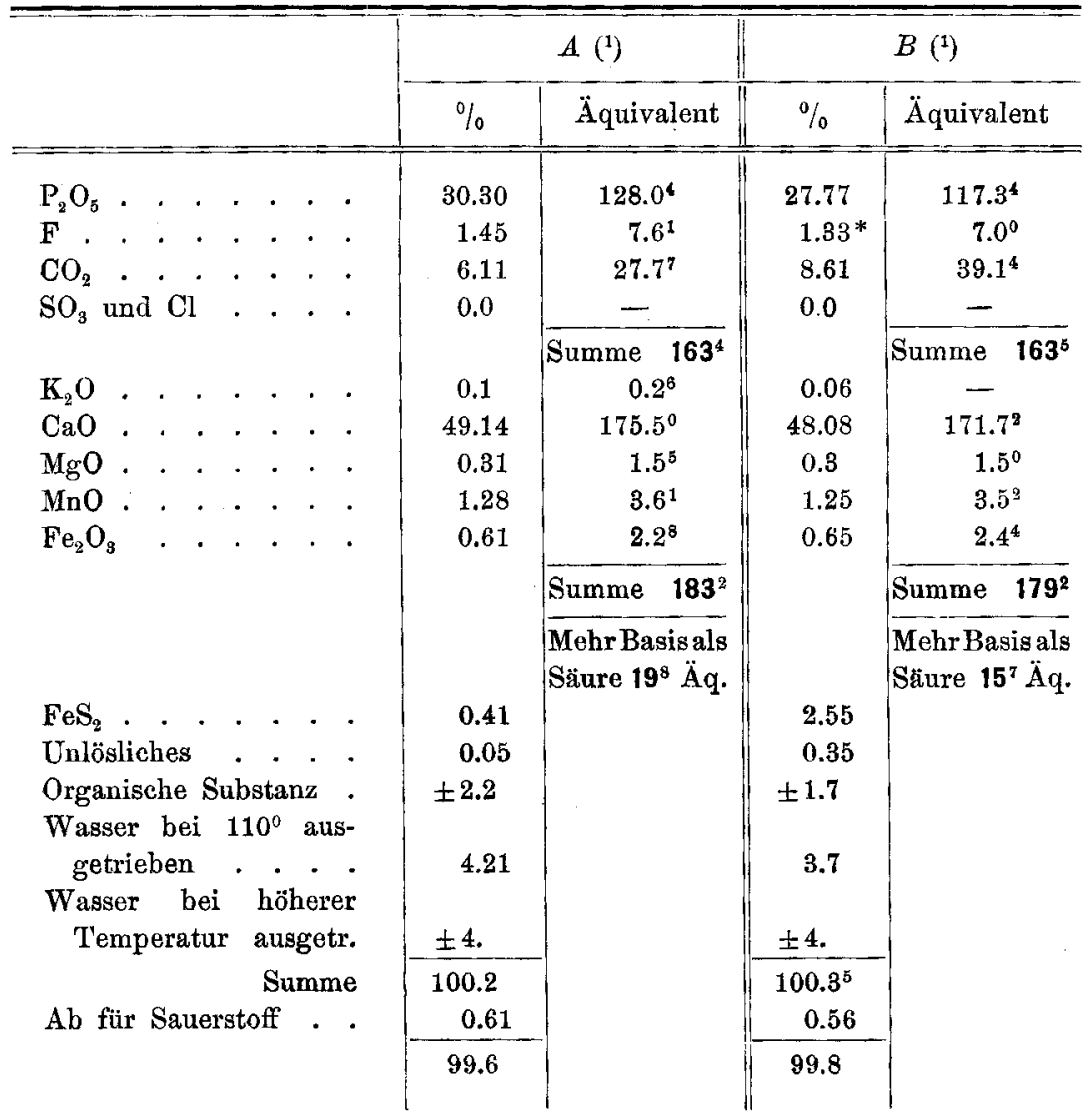

* Berechnet aus dem $\mathrm{P}_{2} \mathrm{O}_{5}$-Gehalt nach dem Verhältnis von $A$.

(1) Die Zahlen sind Mittelzahlen aus zwei oder drei Bestimmungen (siehe darüber den Anhang).

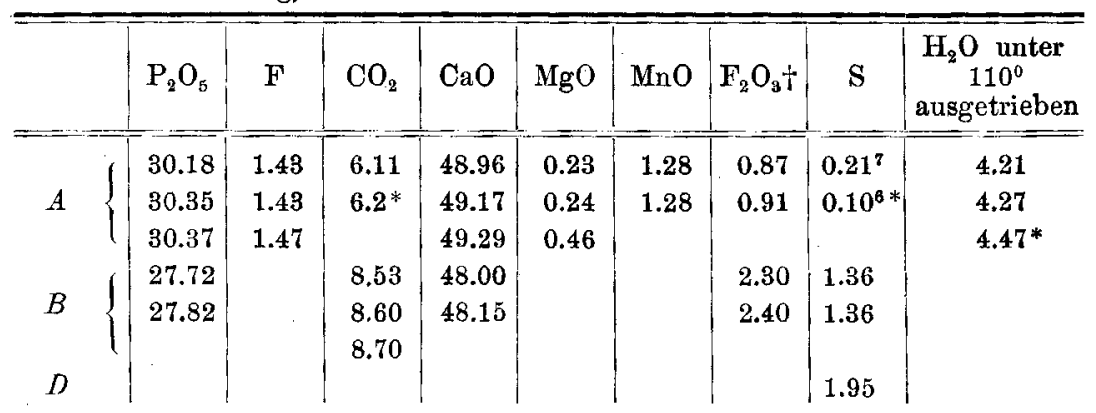

* In einer anderen Portion $A_{1}$.

+ Das sämtliche Eisen als $\mathrm{Fe}_{2} \mathrm{O}_{3}$. 
Aus diesen Zahlen, verbunden mit den Ergebnissen der qualitativen Analyse, lälst sich folgendes ableiten:

Organische Substanz. Die gelbbraune Farbe der Knochensubstanz rührt von dem Überrest des organischen Gewebes her, denn sie tritt mikroskopisch und makroskopisch noch stärker hervor, nachdem durch verdünnte Essigsäure die Karbonate und Phosphate von $\mathrm{Ca}, \mathrm{Mg}$ und $\mathrm{Mn}$ gelöst sind, und nachdem schliefslich auch durch verdünnte Salzsäure der letzte Anteil Phosphat (und alles Eisenoxyd) entfernt ist. Die Menge des organischen Überrestes ist gering. Sie betrug nach den angenäherten Bestimmungen in $A$ höchstens $2.2 \%$, in $B 1.7 \%$, in $C 1.6 \%$ (Siehe unten S. 119), also etwa $1 / 12-1 / 20$ des Gehaltes in der ursprünglichen Knochensubstanz (S. 105). Sie war teilweise löslich in Wasser, weniger in verdünnter Säure. Nach Ausziehen des Karbonats und Phosphats durch verdünnte Essigsäure und Salzsäure blieb nicht ganz 1\% unlösliche Substanz von $A_{1}$ zurück, die sich beim Auswaschen mit Wasser teilweise löste und durch eine verdünnte Säure wieder gefällt wurde - analog den Humussubstanzen.

Die dunkele Farbe der äufseren Schicht rührt nicht von einem gröfseren Eisenoxydgehalt her. Die Analyse dieses Teiles ergab $0.4 \% \mathrm{Fe}_{2} \mathrm{O}_{3}$; indefs in der inneren Schicht $(A) \quad 0.6 \%$ gefunden wurde. Also ist die humöse organische Substanz die Ursache des Farbenunterschiedes. Es ist bekannt, wie humöse Stoffe ihre Farbe von gelb bis schwarz wechseln, womit freilich eine Differenz in dem Gehalt an $\mathrm{C}, \mathrm{H}, \mathrm{O}$ nebenher geht.

Phosphorsäure. Die äquivalente Menge Basen ist gröfser als die äquivalente Menge Säuren; das Phosphat muls also basischer geworden sein, als dem ursprünglichen tertiären Orthosalze des Knochens entspricht. Überdies ist es noch eine Frage, ob die ursprüngliche Menge des Phosphates auch durch Aufnahme von Phosphat aus dem Bodenwasser zugenommen hat, worauf ich unten zurückkomme.

Kalk. Die Äquivalentzahlen machen ersichtlich, dafs auch, wenn alle $\mathrm{P}_{2} \mathrm{O}_{5}, \mathrm{Fl}, \mathrm{CO}_{2}$ als Calciumsalze berechnet werden, noch eine bedeutende Menge $\mathrm{CaO}$ übrig ist und zwar

$$
\begin{array}{ll}
A & \left(175^{5}-163^{4}\right) 28=3.39 \% \mathrm{CaO} . \\
B & \left(171^{\prime}-163^{5}\right) 28=2.3 \% \mathrm{CaO} .
\end{array}
$$

Werden jedoch $\mathrm{MgO}, \mathrm{MnO}, \mathrm{Fe}_{2} \mathrm{O}_{3}$ auch als Phosphat in Rechnung gebracht, dann beträgt der Überschufs an Basis, als Kalk berechnet: 
 \\ A $19.5 \times 28=5.4^{6} \% \mathrm{CaO}$. \\ $B \quad 15.7 \times 28=4.4 \% \mathrm{CaO}$.}

Es sind also nicht allein $\mathrm{MnO}$ und $\mathrm{Fe}_{2} \mathrm{O}_{3}$, sondern auch Kalk aufgenommen, wofür eine Säure fehlt. Dals diese Menge freier Basis an die organische humusartige Substanz gebunden wäre, deren Menge nur $\pm 2 \%$ beträgt, ist gar nicht annehmbar. Wenn auch eine Absorption einer kleinen Menge der Basen durch die Humussubstanz nicht ausgeschlossen ist, so mufs die obige Menge hauptsächlich als ein Bestandteil des Phosphates betrachtet werden.

Magnesia. Sie darf als ein ursprünglicher Bestandteil des Knochens betrachtet werden (siehe S. 106).

Kohlensaurer Kalk. Der Gehalt hat bedeutend zugenommen. Wieviel davon als Ausfüllung der HaFer'schen Kanäle zu betrachten ist (bei $B$ auch in dem geringen spongiösen Teil), wieviel als Ersatz des organischen Gewebes, ist nicht zu bestimmen. Zweifellos ist neu zugekommenes $\mathrm{CaCO}_{3}$ auch in den Lamellen (also zwischen dem Phosphat) eingelagert.

Fluorcalcium. Der Gebalt an $\mathrm{CaF}_{2}$ in den Knochen wurde früher viel zu hoch angenommen. Während CARNoT jetzt nach seiner Methode in den frischen Knochen (Mensch, Seekuh, Rind, Elefant) 0.17-0.3 F findet, so findet $G_{A B R I E L}$ nach seinen sehr sorgfältigen Untersuchungen eine Menge, die unter $0.05 \%$ liegt. ${ }^{1}$ Die Menge Fluor ist also so gut wie gänzlich aus dem Boden aufgenommen. Es ist anzunehmen, dals eine Metamorphose des $\mathrm{CaCO}_{3}$ in $\mathrm{CaF}_{2}$ durch die Alkalifluorüre des Bodenwassers stattgefunden hat. Doch kommt nach meiner Meinung daneben auch eine anziehende Kraft des Phosphates auf das Fluorür ins Spiel, wodurch diese Umsetzung befördert wird. Das Fluorcalcium ist wohl als ein im Phosphat absorbierter Bestandteil zu betrachten.

Eisen und Mangan. Für den Nachweis der Verbindungsform von Eisen und Mangan wurden Stücke von der reinsten kompakten Knochensubstanz $(A)$ ausgelesen, und diese mit jedesmal erneuten Portionen verdünnter Essigsäure $(1: 10)$ behandelt, bis nur Spuren Phosphat sich lösten, nachher mit verdünnter Salzsäure (1:10). Die Untersuchung aller Portionen und des Residuums ergab:

1. dafs Eisenoxydul nur spurenweise anwesend war, und dafs die Essigsäure nur Spuren, die Salzsäure dagegen alles Eisen (aufser dem Pyrit) als Eisenoxyd in Lösung gebracht hat,

1 Siehe die kritische Übersicht der älteren Untersuchungen durch GaBrIEL und seine eigenen Versuche (Zeitschr. physiolog. Chem. (1894) 18, 257-302).

Z. anorg. Chem. XV. 
2. dafs Mangan ${ }^{1}$ nur als Oxydul und in den ersten essigsauren Auszügen vorkam, in den letzten nur spurweise,

3. dafs nach der Behandlung mit Essigsäure durch Salzsäure, neben einer letzten geringen Menge Kalk, Phosphorsäure und dazu Eis en als Eisenoxyd gelöst wurden. Da nun erstens nach der Zufügung von Anmoniak und Schwefelammonium zu dieser salzsauren Lösung (wodurch Calciumphosphat und Schwefeleisen abgeschieden wurden) noch Phosphorsäure in Lösung blieb, zweitens die gelbe Farbe der mit verdünnter Essigsäure ausgezogenen Knochensubstanz durch die Behandlung mit Salzsäure nicht abgeschwächt, sondern nur heller (unter dem Mikroskop) wurde, so lälst sich wohl annehmen, dals das Eisenoxyd als Ferriphosphat anwesend ist. Es ist jedoch die Frage, ob das Eisen nicht $\mathrm{FeO}$ gewesen ist, und nach der Ausgrabung sich an der Luft oxydiert hat. Denn die Abwesenheit von $\mathrm{SO}_{3}$ neben Pyrit beweist, dafs die Reduktionsprozesse im Boden überwiegen. Die Lapillenschicht (unter einem weichen Sandstein), worin der Knochen eingebettet war, befand sich sowohl in der Trocken-wie in der Regenzeit unter dem Wasserspiegel. Weil jedoch das gewöhnliche Vivianit ein Eisenoxyduloxydphosphat ist, das sich an der Luft lange erhält, kommt es mir nicht unwahrscheinlich vor, dals Eisenoxydphosphat im Knochen anzunehmen sein wird.

Das Mangan ist in ziemlich bedeutender Menge $\left(1 \frac{1}{4} \% \mathrm{MnO}\right)$ aufgenommen und zwar als $\mathrm{MnO}$. Der Beweis, dafs es nicht als Karbonat, sondern als Phosphat vorkommt, ist schwerer als für das Eisenoxyd beizubringen. Zwar wird es durch verdünnte Essigsäure gelöst (siehe oben), doch ist 1. Manganphosphat darin löslich, 2. wurde noch etwas Mangan gelöst, nachdem die Kohlensäureentwickelung aufgehört hatte, 3. war nur eine geringe Menge Mangan in den Absätzen des kohlensauren Kalkes in der Axialhöhle des Knochens nachweisbar, 4. kommen in der Natur Manganapatite vor. Dieses alles macht es höchst wahrscheinlich, dafs hier das Mangan nicht, oder nur in geringer Menge als Karbonat in dem Knochen abgesetzt, sondern als $\mathrm{MnO}$ in Phosphat aufgenommen ist.

Pyrit und unlösliche Substanz. Der Pyrit und ein Teil des $\mathrm{CaCO}_{3}$ gehört zu den Einschlüssen. Der Pyrit ist in der ver-

1 Auf Mangan wurde geprüft: 1. mit $\mathrm{PbO}_{2}$ und Salpetersäure, 2. mit Alkalihypochlorit und 3. mit Ferrocyankalium. 
dünnten Säuren unlöslich; als dendritischartiges Gebilde bleibt er also nach dem Ausziehen mit verdünnten Säuren zurück, nebst der organischen Substanz und einer sehr geringen Menge Silikatteilchen. Die letztere Menge betrug nur $0.05 \%$ in $A, 0.35 \%$ in $B$. Die quantitative Bestimmung des Eisens und des Schwefels im ganzen und in dem nach dem Ausziehen mit verdünnten Säuren zurückbleibendem Pyrit bestätigten, dafs Sulfate fehlten, und dafs noch eine gewisse Menge Eisenoxyd als solches in dem in Säuren löslichen Teil vorkam. (Anhang S. 118.)

Chlorüre und Sulfate. Diese kamen nur in unbedentenden Spuren vor.

Kali. Die geringe Menge $0.1 \%$ kann sowohl im Phosphat wie in der Humussubstanz absorbiert sein. Humussubstanzen absorbieren begierig Alkali aus Lösungen freier oder kohlensaurer Alkalien. Ich habe das Kali daher nicht in die Berechnung des Phosphats aufgenommen.

Natron. In dem Auszug mit heifsem Wasser war das Natron nur mikrochemisch nachweisbar.

Wasser. Die Menge Wasser, die über $110^{\circ}$ ausgetrieben wird, kann nur annähernd aus dem Glühverlust bestimmt werden (siehe darüber ausführlich im Anhang S. 119).

Die Analysen von $A$ und $B$ weisen (ohne dieses Wasser) ein Defizit von 4.4 und $4.2 \%$ auf, die Berechnungen aus den Bestimmungen ergeben eine zwischen 3.5 und 4.5 gelegene Zahl. Davon wird nur $\pm 2 \%$ bei der Glühhitze im Rohr der Elementaranalyse ausgetrieben; das übrige beim längeren und stärkeren Glühen, schneller in der Gebläseflamme; noch ein letzter Glühverlust trat ein bei Erhitzung in der Gebläseflamme, als der Stoff mit Kieselsäure vermischt war.

Ich habe also für fossile Knochen, wie kürzlich GabRIEu für frische Knochen gefunden, dals das letzte Wasser erst bei sehr hoher Temperatur ausgetrieben wird. ${ }^{1}$

Dieses Wasser kann kein Bestandteil des kohlensauren Kalkes oder Pyrits sein. Es muls also mit dem Phosphat verbunden sein, und zwar zum Teil so schwach gebunden, dals es mit dem Dampfdruck des überstehenden Wasserdampfes Gleichgewicht hält, teilweise so stark, dafs es erst bei fortwährend steigenden Temperaturen ausgetrieben wird. Von hygroskopischem Wasser (im alten

1 Zeitschr. plyys. Chem. (1894) 18, 257. S. darüber unten (Anh. S. 119, 120). 
Sinne dieses Wortes), Krystallwasser, Hydratwasser u. s. w. darf hier wohl keine Rede sein, nur von der eigentümlichen Bindung des Wassers in amorphen (oder kollordalen) Substanzen, welche eine allgemeine Erscheinung ist.

Nach meiner Ansicht wird das Wasser um so stärker gebunden, als die Menge desselben abnimmt, und der Stoff dabei eine zunehmend höhere Temperatur erhält. Ich betrachte solches als eine Folge der Molenverdichtung, welche die Temperatur in demselben hervorbringt. ${ }^{1}$ Auf diese Sache hoffe ich später zurück zu kommen.

\section{Die Zusammensetzung des Knochens.}

Aus obigen Analysen und den daran geknüpften Betrachtungen folgt, dafs es nicht wünschlich ist, eine prozentische Zusammensetzung nach der herkömmlichen Weise zu berechnen, worin die Salze $\mathrm{P}_{2} \mathrm{O}_{5} .3 \mathrm{CaO}, \mathrm{P}_{3} \mathrm{O}_{5} .3 \mathrm{MgO}, \mathrm{P}_{2} \mathrm{O}_{5} .3 \mathrm{MnO}, \mathrm{P}_{2} \mathrm{O}_{5} . \mathrm{Fe}_{2} \mathrm{O}_{3}$; nebst $\mathrm{CaCO}_{3}$, $\mathrm{CaO}, \mathrm{CaF}_{2}$ u. s. w. figurieren. $\mathrm{Da}$ es jedoch höchst wahrscheinlich ist, dals das Phosphat nebst $\mathrm{CaF}_{2}$ auch basische Oxyde aufgenommen hat, und dals Kohlensäure an Kalk gebunden ist, so ist es besser, die prozentische Zusammensetzung zu berechnen sowie in der folgenden Tabelle geschehen ist. Dabei muls im Auge behalten werden, dafs die Menge des in den HaFER'schen Kanälen eingeschlossenen $\mathrm{CaCO}_{3}$ nicht zu bestimmen ist. In der reinen kompakten Substanz beträgt sie wohl nur den kleineren Teil der gefundenen Menge.

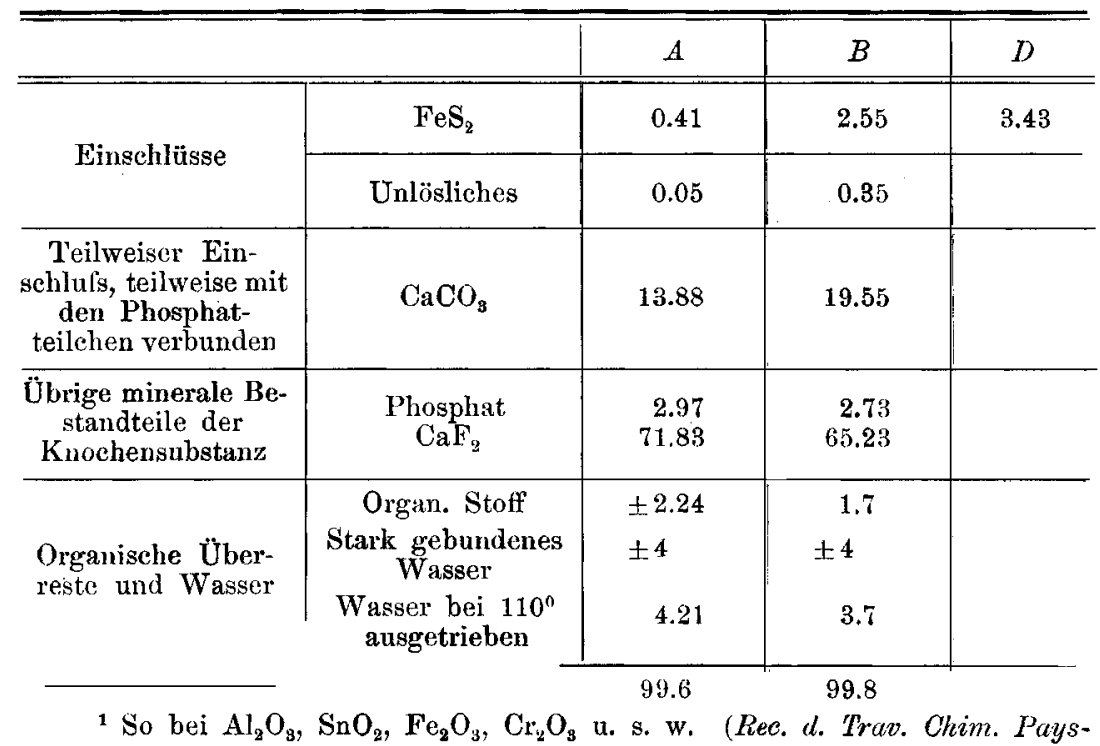

bas. $1889,77,83,96,111,118$. 
Das Phosphat hat die folgende Zusammensetzung ${ }^{1}$ In $A . . \quad . \quad\left[\mathrm{P}_{2} \mathrm{O}_{5}(\mathrm{CaO})^{3}\right]^{3} \cdot 0.53 \mathrm{CaF}_{2} \cdot 1.36 \mathrm{CaO}$.

$$
\begin{aligned}
& \left|\begin{array}{l}
\mathrm{Mg} \\
\mathrm{Mn} \\
\mathrm{Fe}^{2} / \mathbf{s}
\end{array}\right| \\
& \text { In } B \text {. . }\left[\mathrm{P}_{2} \mathrm{O}_{5}(\mathrm{CaO})^{3}\right]^{3} \cdot 0.54 \mathrm{CaF}_{2} \cdot 1.19^{5} \mathrm{CaO} \text {. } \\
& \left|\begin{array}{l}
\mathrm{Mg} \\
\mathrm{Mn} \\
\mathrm{Fe}^{2} / \mathrm{s}
\end{array}\right|
\end{aligned}
$$

Die molekularen Mengen der Monoxyde betragen:

\begin{tabular}{ll}
$A$ & \multicolumn{1}{c}{$B$} \\
$0.85 \mathrm{CaO}$ & $0.63 \mathrm{CaO}$ \\
$0.11 \mathrm{MgO}$ & $0.11^{5} \mathrm{MgO}$ \\
$0.25 \mathrm{MnO}$ & $0.27 \mathrm{MnO}$ \\
$0.15\left(2 / \mathrm{Fe}_{2} \mathrm{O}_{9}\right)$ & $0.18\left({ }^{2} / \mathrm{Fe}_{2} \mathrm{O}_{3}\right)$ \\
\hline 1.36 & $1.19^{5}$
\end{tabular}

Das Phosphat hat also dieselbe Zusammensetzung in $A$ und $B$. $B$ enthält mehr $\mathrm{CaCO}_{3}$ und $\mathrm{FeS}_{2}$. Aufserdem kann dazu noch basisches Wasser gerechnet werden; wieviel, ist jedoch nicht angebbar. Die ganze Menge Wasser, auf 1 Mol. Phosphat berechnet, beträgt:

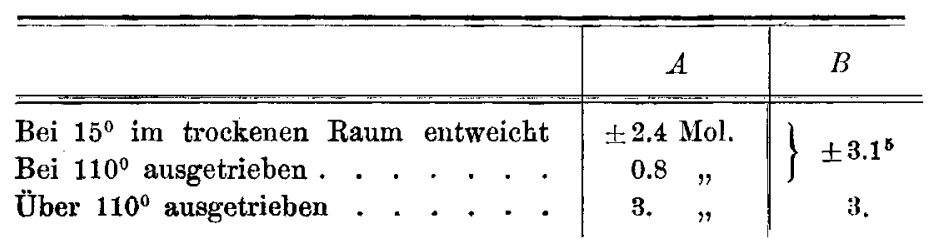

Es sind also $\pm 3 \%$ als schwach absorbiertes und wenigstens $3 \mathrm{Mol}$. als stärker absorbiertes Wasser auf 1 Mol. Phosphat anwesend. für $B)$ :

Diese Formel ist auf die folgende Weise berechnet für $A$ (wie auch

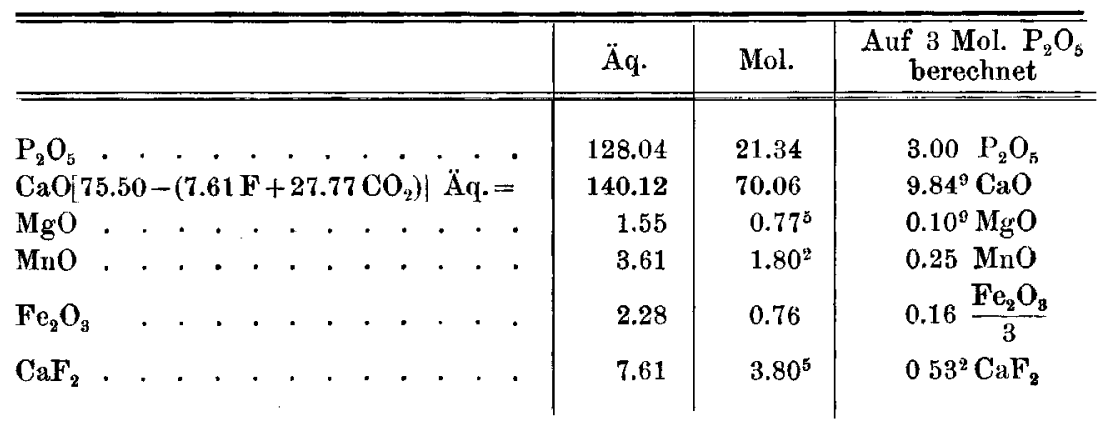


Die $2 \%$ organische Substanz kann nur einen kleinen Teil davon gebunden halten. Also mufs es gröfstenteils im Phosphat absorbiert sein.

Sollte man annehmen (was jedoch unwahrscheinlich ist, S. 98), dals das Mangan ganz oder teilweise als Karbonat vorkäme, so würden sich die Zahlen 1.36 und $1.19^{5}$ in der obigen Formel nicht ändern; denn in diesem Falle muls die äquivalente Menge Kalk um ebensoviel vermehrt werden, wie die Menge Mangan im Phosphat verringert wird. ${ }^{1}$

Es ist beachtenswert, dafs kalte verdünnte Essigsäure nur sehr langsam das Phosphat löst, und dafs das Phosphat noch dieselbe basische Zusammensetzung aufweist, nachdem der grölste Teil gelöst war. Als die Substanz verschiedene Male nach einander mit verdünnter Essigsäure ausgezogen war, bis ungefähr $1 / 5 \mathrm{des}$ Phosphats übrig geblieben, enthielt dieser Rückstand $6.31 \% \mathrm{P}_{2} \mathrm{O}_{5}$, $8.52 \% \mathrm{CaO}$ und $0.4 \% \mathrm{Fe}_{2} \mathrm{O}_{3}$ (mit einer Spur Mangan) in Salzsäure löslich (bezogen auf die ursprüngliche Menge). Die Zusammensetzung dieses Phosphates war also:

$$
\left[\mathrm{P}_{2} \mathrm{O}_{5} .3 \mathrm{CaO}\right]^{\mathrm{s}} \begin{cases}1.26 & \mathrm{CaO} \\ 0.15 & \mathrm{Fe}_{2} \mathrm{O}_{3}\end{cases}
$$

welche Formel wenig von der obigen verschieden ist.

\section{Die Änderungen, die der Knochen erlitten hat.}

a. Die zugekommenen Bestandteile.

Herr Dubors hat mich darauf aufmerksam gemacht, dals die in der oben erwähnten Tertiärschicht gefundenen fossilen Knochen ein höheres spez. Gewicht als frische Knochen besitzen, und dafs sich daraus berechnen lälst, um wieviel der ursprüngliche Gehalt an jedem der Bestandteile zu- oder abgenommen hat. Er hatte die Güte, mir folgende Zahlen mitzuteilen.

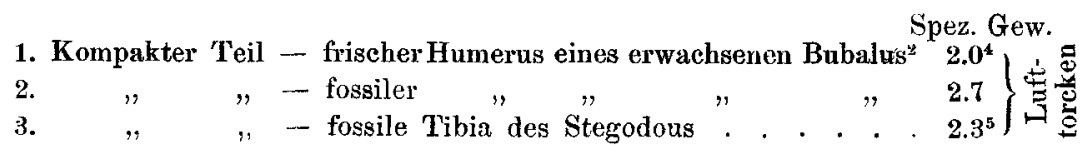

${ }^{1}$ Dasselbe gilt für die Magnesia. Wiurde das Eisen als froies $\mathrm{Fe}_{2} \mathrm{O}_{3}$ berechnet, was jedoch nicht anzunehmen ist, so wäre die Menge basischen Oxyds etwas kleiner: 1.20 und 1.01 .

Büffel, javanischer Bubalus suec. 
Der fossile Bubalus ist nur eine Varietät von der noch auf Java lebenden Art. Der fossile Stegodon ist wahrscheinlich ein nicht ganz erwachsenes Tier gewesen. Herr Dubors hat die reinsten Stücke der kompakten Substanz, frei von Pyrit und Einschlüssen, für seine Bestimmungen gewählt. ${ }^{1}$

Ich fand unter den nämlichen Versuchen für die kompakte Substanz des analysirten Stegodonknochens in zwei Bestimmungen spez. Gewicht: 2.2. Das spez. Gewicht von frischen Knochen ist nur selten bestimmt; es wird gewöhnlich auf 1.8-2.0 angegeben. AEBY $^{2}$ fand für Rind, Pferd, Affen \pm 2.05 , für den Mensch 1.9-1.96. Andere Bestimmungen habe ich nicht gefunden. Nur Shari'tes ${ }^{3}$ giebt an 2.07 für den frischen Knochen eines Manatus australis (Seekuh) und für einen fossilen 2.8 ${ }^{3}$. GöвEL ${ }^{4}$ für einen fossilen Knochen von Rhytina Stelleri ${ }^{5} 2.18$ und 2.21. Die Zahlen von Sharples sind fast die nämlichen wie die von Dubois für den Bubalus. Die oben erwähnte Berechnung aus dem spez. Gewicht ist nur erlaubt, wenn der fossile Knochen bei der allmählichen Verwesung des Osseins und der Aufnahme von Kalksalzen u. s. w. keine Zusammenziehung erfährt. Diese ist jedoch nie beobachtet. Der anatomische Bau bleibt derselbe (Seite 91). Die Palaeontologen und Anthropologen haben immer angenommen, dals fossile Knochen ihre Gröfse und Form ungeändert behalten. Bei fossilen und diluvialen Menschenknochen ist das immer beobachtet. So scheint es denn wohl erlaubt, die Berechnung der zugekommenen Bestandteile auf das spez. Gewicht zu gründen.

Die Porosität der kompakten Substanz in unseren Knochen ist gering, nicht grölser als $1 / 12$ seines Volums. Der rom Osseingewebe eingenommene Raum ist durch Kalksalze ausgefüllt. Das erklärt also genügend die Vergrölserung des spez. Gewichtes, welches bei den erwachsenen Bubalus bis 2.7 (übereinstimmend mit Sharples, Beobachtung des Manatus), b\&i dem wahrscheinlich nicht erwachsenen Stegodon bis 2.2-2.3 gestiegen ist. Das spez. Gewicht des Osseins ist geringer als vom $\mathrm{CaCO}_{3}(2.7-2.9)$ und vom $\mathrm{Ca}_{3} \mathrm{P}_{2} \mathrm{O}_{8}(3.2)$.

1 Bestimmung mit der Westruar'schen Wage. Die Stücke wogen 8.61 und $10.27 \mathrm{~g}$ und wurden mit einer dünnen Firnissehicht überzogen.

${ }^{2}$ Centralbl. Med. Wiss. (1871) 9, 210, 561 und 1098.

3 Amer. Journ. [Sill.] (1871) [3] 1, 168.

4 Journ. pr. Chem. (1862) 86, 318.

s Das Borkentier, im vorigen Jahrhundert ausgerottet, eine pflanzenfressende Cetacea. 
Die Zusammensetzung der Knochen, auch wenn man nur die langen Knochen betrachtet, ist bei mehreren Tieren verschiedenen Alters eine andere, was den Gehalt an organischer Substanz, Wasser, Calciumkarbonat, Calciumphosphat anbetrifft. Dazu kommt, dals die meisten Analysen nur die Asche betreffen, und dafs die Genauigkeit zu wünschen übrig lälst, besonders die Bestimmung des organischen Teiles und der Kohlensäure. Die Bestimmung des Wassers läfst alles zu wünschen übrig. Jedoch kann man einen Maximum-, Minimum- und Mittelwert annehmen von 32, 28, 30\% Ossern. In der folgenden Tabelle ist die Aschenanalyse eines Elefantenknochen von $\mathrm{CARNOT}^{1}$ sowohl auf 32 , wie auf $28 \%$ organische Substanz berechnet; daneben ist eine von GabrigL's ${ }^{2}$ Analysen (auf

\begin{tabular}{|c|c|c|c|c|c|c|}
\hline & I. & II. & \multicolumn{2}{|c|}{ III. } & IV \\
\hline & & \multicolumn{2}{|c|}{$\begin{array}{l}\text { Femur eines Elefanten } \\
\text { (Camor) }\end{array}$} & \multicolumn{2}{|c|}{$\underset{\text { (GABRIEL) }}{\operatorname{Rind}}$} & $\begin{array}{l}\text { Eine mittlere } \\
\text { Zusammen- } \\
\text { setzung }\end{array}$ \\
\hline $\begin{array}{l}\text { Organisch } \\
\mathrm{Ca}_{3} \mathrm{P}_{2} \mathrm{O}_{8} \cdot \\
\mathrm{Mg}_{9} \mathrm{P}_{2} \mathrm{O}_{8} \cdot \\
\mathrm{CaCO}_{8} \cdot \cdot \\
\text { Übrige Bestand }\end{array}$ & $\begin{array}{l}\cdot \\
\cdot \\
\cdot \\
\cdot \\
\dot{e} \\
\text { eile. }\end{array}$ & $\begin{array}{l}32 .- \\
61.2 \\
1.3^{3} \\
4.9^{4} \\
0.5^{3}\end{array}$ & $\begin{array}{c}28 .- \\
64.8^{-} \\
1.4^{1} \\
5.2^{3} \\
0.5^{6}\end{array}$ & & & $\begin{array}{l}30 .- \\
61 .- \\
1^{3} \\
7^{2} \\
0^{5}\end{array}$ \\
\hline & & $100 .-$ & $100 .-$ & & & $100 .-$ \\
\hline \multirow[t]{3}{*}{ Also an $\mathrm{P}_{2} \mathrm{O}_{5}$} & . & $28.7^{2}$ & 3.04 & \multicolumn{2}{|c|}{$26.7^{3}$} & $28.6^{7}$ \\
\hline & \multicolumn{3}{|c|}{$\begin{array}{l}{ }^{1} \text { CARNoT, Compt. rend. (1892) } \\
\text { 114, 1189. }\end{array}$} & \multicolumn{3}{|c|}{$\begin{array}{l}{ }^{2} \text { GABRIEL, Zeitschr.phys. Chem. } \\
\text { (1893) 18, 281. }\end{array}$} \\
\hline & Elefant & Mensch & Rind & Mensch & Rind & Ganze \\
\hline \multirow[t]{2}{*}{ 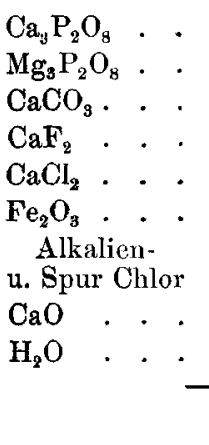 } & $\begin{array}{r}90 .- \\
1.96 \\
7.27 \\
0.47 \\
0.20 \\
0.15\end{array}$ & $\begin{array}{r}87.45 \\
1.57 \\
10.18 \\
0.35 \\
0.23 \\
0.10\end{array}$ & $\begin{array}{r}85.72 \\
1.53 \\
11.96 \\
0.45 \\
0.30 \\
0.13\end{array}$ & $\begin{array}{c}77.5 \\
1.69 \\
13.30 \\
<0.1 \\
- \\
- \\
1.37 \\
1.89 \\
2.46\end{array}$ & $\begin{array}{c}79.06 \\
2.30 \\
11.5 \\
<0.1 \\
- \\
- \\
1.31 \\
2.00 \\
2.33\end{array}$ & $\begin{array}{c}80.08 \\
2.78 \\
9.34 \\
<0.1 \\
- \\
- \\
1.30 \\
2.38 \\
3.05\end{array}$ \\
\hline & 100.05 & 99.88 & 100.09 & 98.2 & 98.5 & 98.93 \\
\hline
\end{tabular}


$30 \%$ organische Substanz berechnet), und eine mittlere Zusammensetzung der Knochen gegeben. ${ }^{1}$

Wenn wir nun für das ursprüngliche spez. Gewicht des Stegodonknochens 2.0 annehmen und im fossilen Zustand 2.2, so ist das Gewicht eines gleichen Volums von 1 auf 1.1 vermehrt; nach der Bestimmung von DuboIs auf 1.15 ; bei dem Bubalus bis auf 1.35. Im ersten (niedrigsten) Falle, und bei der Annahme, dafs die organische Substanz bis auf $2.5 \%$ verschwunden ist:

\begin{tabular}{|c|c|c|c|c|}
\hline & I. & II & III. & IV. \\
\hline $\begin{array}{l}\text { Von } 100 \text { ursprünglichen Bestand- } \\
\text { teilen übrig geblieben } . \cdot \text {. }\end{array}$ & $70^{5}$ & $74^{5}$ & $72^{5}$ & $72^{5}$ \\
\hline \multirow[t]{2}{*}{ Also zugekommen } & $39^{5}$ & $35^{5}$ & $37^{5}$ & $37^{5}$ \\
\hline & 110 & 110 & 110 & 110 \\
\hline
\end{tabular}

Woraus für die Zusammensetzung auf 100 folgt:

\begin{tabular}{|c|c|c|c|c|c|c|}
\hline & I. & II. & III. & IV. & \begin{tabular}{|} 
Analyse d. \\
Stegodon- \\
knoch. $(A)$ \\
$($ Tb. S. 95)
\end{tabular} & $\begin{array}{c}\text { Also } \\
\text { zugekommen } \\
\text { oder ver- } \\
\text { schwunden }\end{array}$ \\
\hline \multirow{8}{*}{$\begin{array}{l}\text { Organisch . } \\
\mathrm{Ca}_{8} \mathrm{P}_{2} \mathrm{O}_{8} \\
\mathrm{Mg}_{3} \mathrm{P}_{2} \mathrm{O}_{8} \\
\mathrm{CaCO}_{8} \cdot \\
\mathrm{CaO} . \\
\text { Übrige Be- } \\
\text { standteile. } \\
\text { Zugekom- } \\
\text { men . . }\end{array}$} & $2.2^{4}$ & $2.2^{4}$ & $2.2^{4}$ & $2.2^{4}$ & $2.2^{4}$ & - \pm 28 \\
\hline & $55.6^{5}$ & $58.9^{1}$ & 50.9 & $55.4^{6}$ & 65.3 & $+6^{5}-14$ \\
\hline & $1.2^{1}$ & $1.2^{8}$ & $1.7^{5}$ & 1.2 & $0.6^{7}$ & -0.5 \\
\hline & 4.5 & $4.7^{5}$ & $5.9^{1}$ & $6.5^{4}$ & $13.8^{5}$ & $+7-9$ \\
\hline & - & - & $1.8^{2}$ & - & $3.8^{1}$ & $+2-4$ \\
\hline & $0.4^{8}$ & $0.5^{1}$ & $3.2^{*}$ & $0.4^{8}$ & $14.1^{3}$ & $\begin{cases}3.0 \mathrm{CaF}_{2} \\
1.3 \mathrm{MnO}\end{cases}$ \\
\hline & $35.9^{8}$ & $32.3^{1}$ & $34.1^{8}$ & 34.1 & - & ${ }^{+} \mid \begin{array}{ll}0.6 & \mathrm{Fe}_{2} \mathrm{O}_{3} \\
0.4 & \mathrm{FeS}_{2}\end{array}$ \\
\hline & 100 & 100. & 100.- & 100 & $100 .-$ & \\
\hline $\mathrm{P}_{2} \mathrm{O}_{5}$ & $26.1^{4}$ & $27.6^{7}$ & $24.2^{3}$ & $26.0^{8}$ & 30.3 & $+2.7-6$ \\
\hline
\end{tabular}

* Durch Gabrigl gefundenes Wasser und Alkalien.

Aus früheren Analysen von fossilen Knochen läfst sich diese Berechnung nicht ausführen, weil die nötigen Daten fehlen. Nur

1 Ich habe hierzu die Analysen von Frerichs, Zaleskx, von Brbra, HoppeSeyler, Milne-Edwards, Schützengerger, Aeby u. s. w. benutzt. 
Sharples Analyse erlaubt eine derartige Berechnung ${ }^{1}$ und ergiebt die bedeutende Zunahme von $12.9 \% \cdot \mathrm{P}_{2} \mathrm{O}_{5}{ }^{2}$

Die Analyse einer grölseren Zahl von fossilen Knochen aus verschiedenen geologischen Perioden ist erwünscht, um die Aufnahme von Phosphorsäure sicher zu stellen.

Aus dem spez. Gewicht und der Analyse werden also für die reine kompakte Knochensubstanz $A$ als von aufsen zugekommen berechnet (siehe die letzte Spalte in der vorigen Tabelle):

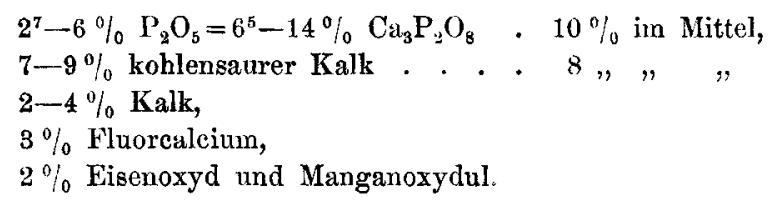

Die Magnesia ist dagegen anf die Hälfte vermindert. In Substanz $B$ ist noch mehr kohlensaurer Kalk, und eine Menge von 2.55 Pyrit eingelagert; in $D 3.5 \%$ Pyrit. Zieht man in Betracht: 1. dafs bei I-IV sicherlich noch einige Prozente Wasser zugerechnet werden müssen, wodurch ihre Zahlen etwas niedriger werden, 2. dafs ein ursprünglicher Gehalt von $32 \%$ organischer Substanz viel wahrscheinlicher ist, als von $28 \%$, so stellt sich heraus, dafs die obige Mittelzahl wohl nicht

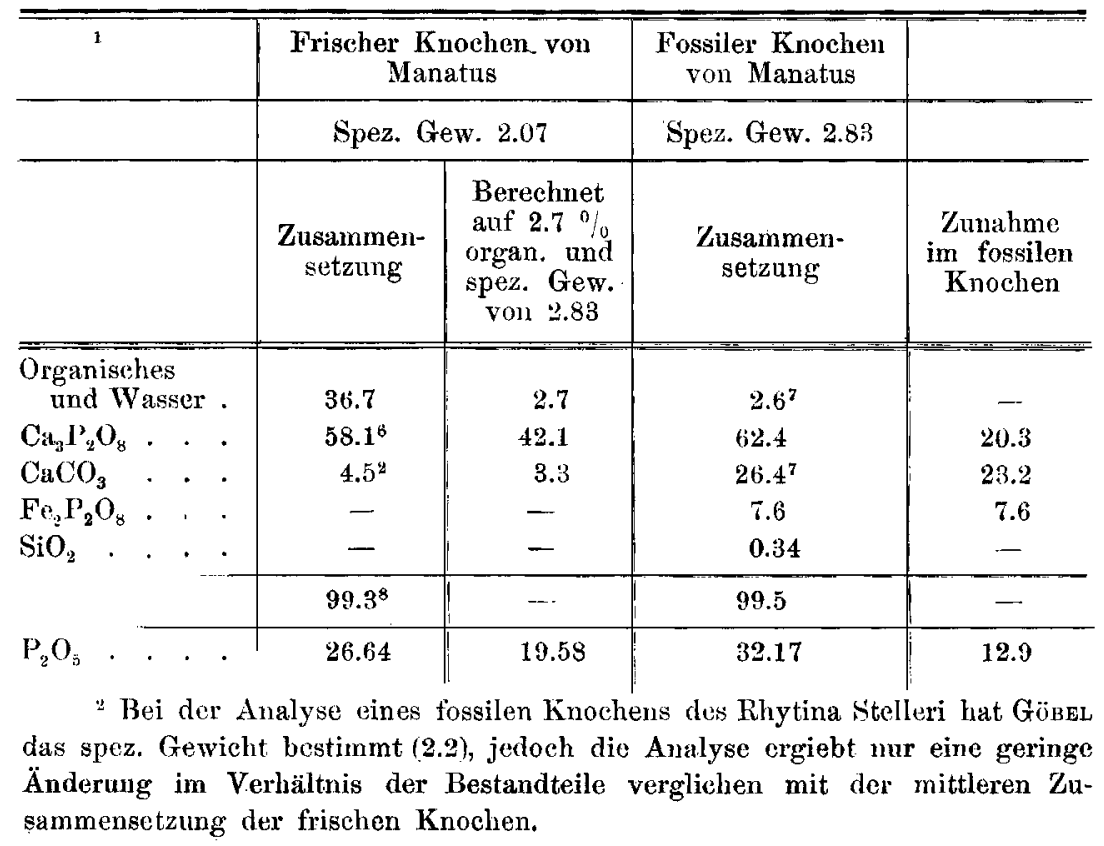


zu hoch gegriffen ist. ${ }^{1}$. Bei einem spez. Gewicht von $2.3^{5}$ (DUbors) kann die Zunahme $3^{8}-7 \quad \mathrm{P}_{2} \mathrm{O}_{5}=9-16^{6} \mathrm{Ca}_{3} \mathrm{P}_{2} \mathrm{O}_{8}$ betragen.

Es ist also nicht allein Karbonat, sondern auch Phosphat aufgenommen, und wir können annehmen, dafs nicht allein die Kanäle sich mit $\mathrm{CaCO}_{3}$ ausgefüllt haben, sondern dafs 1. die Osseïnteilchen der Lamellen durch einen Komplex von $\mathrm{Ca}_{3} \mathrm{P}_{2} \mathrm{O}_{8}, \mathrm{CaCO}_{3}, \mathrm{CaF}_{2}, \mathrm{CaO}$ ersetzt sind, 2, die Hälfte des $\mathrm{Mg}_{3} \mathrm{P}_{2} \mathrm{O}_{8}$ in $\mathrm{Ca}_{3} \mathrm{P}_{2} \mathrm{O}_{8}$ (oder in $\mathrm{Mn}_{3} \mathrm{P}_{2} \mathrm{O}_{8}$ ) metamorphosiert ist, und sich aufserdem $\mathrm{Fe}_{2} \mathrm{P}_{2} \mathrm{O}_{8}$ und $\mathrm{Mn}_{3} \mathrm{P}_{2} \mathrm{O}_{8}$ gebildet haben.

\section{b. Die Absetzungen und Umsetzungen.}

Die Ergebnisse der Analyse stimmen zu einem Teil mit denen der älteren Untersuchungen von fossilen Knochen überein ${ }^{2}$ und geben aufserdem zu einigen neuen Gesichtspunkten Veranlassung.

Das Osseyn des Knochens ist bis zu einem humusartigen Rest (von 2-2.5\%) verschwunden. Die HAFER'schen Kanäle und die in dem spongiösen Knochenteil entstandenen Hohlräume sind mit krystallinischem $\mathrm{CaCO}_{3}$ ausgefüllt; darum enthält Substanz $B$ fast $6 \%$ mehr als $A$. Der in den Lamellen des kompakten Teiles durch das Verschwinden der organischen Substanz entstandene Raum ist,

${ }^{1}$ Selbst wenn ein niedriger Gehalt an Osseïn und Wasser $(28 \%$ ), und ein sehr hoher Gehalt an $\mathrm{P}_{2} \mathrm{O}_{5}(90 \%$ im Elefantenknochen - CARNoT - ) angenommen werden, lälst sich noch eine Zunahme von etwa $3 \% \mathrm{P}_{2} \mathrm{O}_{5}$ berechnen.

2 Die Änderungen, welche die Analysen von fossilen Knochen bis jetzt angezeigt haben, sind: 1. Abnahme der organischen Substanz, im allgemeinen von dem geologischen Alter abhängig; 2. Abnahme der Magnesia; 3. Abnahme oder Zunahme des kohlensauren Kalks; 4. Aufnahme von $\mathrm{CaF}_{2} ; 5$. Aufnahme in einigen Fällen von $\mathrm{Fe}_{2} \mathrm{O}_{3}, \mathrm{MnO}, \mathrm{Na}_{2} \mathrm{O}$, Sulfaten, $\mathrm{SiO}_{2}$ : Die meisten AnaIysen scheinen, in Hinsicht der Bestandteile unter 5. genannt, unvollständig zu sein. Pyrit wird nicht erwähnt. Eine Zunahme des phosphorsauren Kalks ist nicht bemerkt. Axby erwähnt, dafs in den Knochen der Pfahlbauten $\mathrm{FeCO}_{\mathrm{g}}$ und $\mathrm{MnCO}_{3}$ als metamorphosiertes $\mathrm{CaCO}_{3}$ eingelagert sind, und dals sieh ein Teil des Calciumphosphats in Vivianit umsetzt. Das $\mathrm{CaF}_{2}$ ist nach ihm entstanden aus $\mathrm{CaCO}_{3}$ durch Einwirkung von gelösten Fluorüren. Was die $\mathrm{Ab}$ oder Zunahme des $\mathrm{CaCO}_{3}$ im fossilen Knochen anbetrifft, scheint sie nicht allein von den Bodenverbältnissen, sondern auch von dem Alter der Bodenschicht abhängig zu sein. AfBy beobachtete eine Abnahme bei den Knochen der Pfahlbauten bis auf $1 \%$. Ebenso $W_{\text {IBEL }}$ (Ber. deutsch. chem. Ges. [1874] 7, 23). Delesse (Compt. rend. [1861] j2, 729) bemerkt, dals das $\mathrm{CaCO}_{3}$ in den jüngsten Absetzungen (quaternären) abgenommen, in den älteren zugenommen hat. Er leitet das Alter des Knochens aus dem Stickstoffgehalt ab, der nach ihm bei den älteren vortertiären Knochen ganz verschwunden ist. 
wenn die Berechnungen von S. 105 als richtig betrachtet werden, durch neu hinzugekommenes Calciumphosphat und durch Kalk wieder ausgefüllt, so dafs das spez. Gewicht auf 2.2-2.3 gestiegen ist.

Durch einen bekannten Reduktionsprozels hat sich in den Kanälchen des Gewebes Pyrit gebildet ${ }^{1}$ und zwar 1. in der äufseren Schicht des kompakten Teiles bis zu einer gewissen Tiefe, 2. in dem Übergange zum spongiösen Gewebe, 3. in den Markhöhlen. Die mittlere Schicht des kompakten Teiles ist noch frei oder fast frei von Pyrit.

Die Metamorphosen, die stattgefunden haben, sind folgende: 1. eine Umsetzung von etwa $3 \% \mathrm{CaCO}_{3}$ mit den Fluorüren des Bodenwassers zu $\mathrm{CaF}_{2}$, welches ohne $\mathrm{Z}_{\text {weifel auf eigentümliche } \mathrm{Weise}}$ mit dem Phosphat verbunden ist, 2. eine Umsetzung einer kleinen Menge Magnesiumphosphat - und dazu einer gewisse Menge Calciumphosphat - mit den Mangan- und Eisensalzen des Bodenwassers zu $\mathrm{Mn}_{3} \mathrm{P}_{2} \mathrm{O}_{8}$ und $\mathrm{Fe}_{2} \mathrm{P}_{2} \mathrm{O}_{8}$. Die Magnesia ist dadurch verringert; sie ist merkwürdigerweise nicht aus dem Bodenwasser aufgenommen. Zuletzt ist noch, ohne Substitution, eine nicht unbedeutende Menge Kalk im Phosphat aufgenommen, denn die Basen übertreffen in $A$ die Säuren um ungefähr $1 / 8$ ihres Betrages (in $B$ um 1/10); auf $1 \mathrm{Mo}$ lekül Phosphat ist fast $1 / 2$ Molekül Basis im Überschufs vorhanden. Diese letzte Erscheinung erfordert jedoch eine nähere Betrachtung, weil in der letzten Zeit wieder die Frage aufgeworfen worden ist, ob nicht das Phosphat schon im frischen Knochen basisch ist.

In den älteren Untersuchungen, von welchen nur die kleinste Zahl die unverbrannten Knochen betrifft, ist von einem Überschuls von Basis in den frischen Knochen nie die Rede gewesen. Jedoch 1894 hat GABRIEL ${ }^{2}$ solches aus sehr genauen Analysen abgeleitet. Sie beträgt $1 / 20-1 / 15$ der Basisäquivalente, was einer Menge von $3 \%$ Kalk entspricht, so dafs die Zusammensetzung des Phosphates dargestellt wird durch die Formel $\left(\mathrm{Ca}_{3} \mathrm{P}_{2} \mathrm{O}_{8}\right)_{5} \cdot \mathrm{Ca}(\mathrm{OH})_{2} \pm 2 \mathrm{H}_{2} \mathrm{O}$. GABRIEL hat eine neue Methode angewandt, um die Knochen vollständig von Ossern zu befreien, nämlich durch Erhitzung derselben bei $150^{\circ}$ mit 2.5\% kalihaltigem Glycerin. Die Kohlensäure ist jedoch nicht im ursprünglichen Knochen, sondern nach dieser Behandlung bestimmt. Daher bleibt es einigermalsen zweifelhaft, ob vielleicht das Kali dem Knochen etwas Säure entzogen hat. Auch ist zu be-

1 Über das Vorkommen und die Bildung von Pyrit und Schwefel im moorigen Boden, siehe meine Untersuchung in den Abhandl. der kgl. Akad. der Wissensch. Amsterdam 1886 (S. 50-67).

2 Journ. physiolog. Chem. (1893) 18, 257-304. 
merken, dals GaBRIEL mehr Alkalien in den Knochen gefunden hat, als die :̈̈lteren Untersuchungen angegeben haben. ${ }^{1}$

Neue Bestimmungen der Kohlensäure und des Verhältnisses zwischen Säuren und Basen in frischen Knochen sind also sehr erwünscht.

Bei fossilen Knochen ist ein Kalküberschuls erst von AEBY konstatiert, nämlich $4.28 \% \mathrm{CaO}$ in einem Menschenknochen, der noch die Hälfte seiner organischen Substanz enthielt und $5.27 \%$ in einem Zahnbein, das ganz frei war von organischer Substanz (aus den Pfahlbauten der Schweiz). Aus den übrigen Analysen unverbrannter Knochen lälst sich nichts Sicheres ableiten. ${ }^{2}$ Jedoch aus

1 Gabrier hat $1.0-1.1 \% \mathrm{Na}_{2} \mathrm{O}$ und $0.2-0.3 \mathrm{Kali}$ ( \pm 4 Äq.) gefunden, obgleich das feine Knochenpulver vorher mit Wasser, Alkohol, Äther ausgezogen war. In der obigen Formel des Phosphats ist dann auch ein Teil des Kalks durch $\mathrm{Na}_{2} \mathrm{O}$ und $\mathrm{K}_{2} \mathrm{O}$ ersetzt. Die Berechnung ergab:

\begin{tabular}{c|c|c|c}
\hline Aquivalent & $\begin{array}{l}\text { Mensch. } \\
\text { Oberarm- } \\
\text { knochen }\end{array}$ & $\begin{array}{c}\text { Rind. } \\
\text { Schenkel- } \\
\text { knochen }\end{array}$ & $\begin{array}{c}\text { Gänse. } \\
\text { Sämtliche } \\
\text { Knochen }\end{array}$ \\
\hline Basen . - . & $191^{1}$ & $192^{3}$ & $192^{5}$ \\
Säuren .- & $181^{4}$ & $181^{3}$ & 180 \\
\hline Differenz: & $9^{7}$ & 11 & $12^{5}$
\end{tabular}

Kann das Phosphat etwas Kali aus dem kalihaltigen Glycerin absorbiert haben und trotz des Auswaschens zurückhalten?

${ }^{2}$ AxBP bestimmte richtigerweise die Kohlensäure in der unverbrannten Substanz. Was die übrigen Analysen der fossilen Knochen anbetrifft, so hat v. Brara (Chem. Unters. d. Knochen des Menschen u. d. Wirbeltiere. Schweinfurt 1844), der ebenso die Kohlensäure in den unverbrannten Knochen bestimmte, behauptet, dafs er bei seinen zahlreichen Analysen nie ein Defizit an Säure erhalten hat (ebenda S. 97). Da er seine Zahlen für frische wie für fossile Knochen alle auf 100 umgerechnet hat, so entziehen sie sich jeder Kontrolle. Auch FrEmy (Ann. Chim. Phys. [1855] 43, 88), der nur die Zahlen der berechneten Salze angiebt, hat kein Defizit bei seinen Analysen von fossilen Rhinozeros-, Hyaena-, Ursusknochen bemerkt; doch sind seine Analysen nicht genau. Sharples (siehe oben) giebt die Säuren und Basen nicht getrennt an; ebensowenig Garnier in Frúmy's Encyklopädie für die Analysen eines Humerus und Femurs des Ursus spelaeus dureh Krocker und dureh GAUTIER (Chimie physiol. Liquides et Tissus 9, 606). Die Aschenanalyse der Wirbelknochen eines fossilen Walfisches, des Zeuglodons (Baumert, Journ. pr. Chem. [1851] 54, 363) ergab ein Mehr an Basis. Lenmann (ebenda) erhielt dagegen ein Mehr an Säure bei der Rippe eines fossilen Hydrarchos, doch ist der Gehalt an Fluor (8.05) sicher viel zu hoch gefunden. GreEnE's Knochenanalysen 
den Analysen von AkBy und den meinigen folgt, dafs dieser Uberschuls an Kalk (oder im allgemeinen an Basis) in den fossilen Knochen vorkommt. Wenn auch die Analyse von GABRIEL durch neuere Untersuchungen von frischen Knochen bestätigt wird, so ist doch die Menge überschüssiger Basis in den fossilen Knochen des Stegodons viel grölser, als die durch GaBRIEL in frischen Knochen gefundene.

\section{Die Beschaffenheit (chemische) des Phosphats.}

Welcher Art ist das Phosphat, aus dem reinen kompakten Teil", frei von Einschlüssen, das teilweise zu Mangan- und Ferriphosphat metamorphosirt ist, und $\mathrm{CaFl}_{2}, \mathrm{CaCO}_{3}$ in sich aufgenommen hat? Welche chemische Beschaffenheit darf man diesem Komplex zuschreiben?

Man könnte die Ansicht hegen, dal's er eine langsame Umwandlung in Apatit darstellt.

Also von welcher Art muls nun diese Verbindung gehalten werden?

In der Natur kommen Apatitkrystalle vor, worin ein Teil des Fluors durch Chlor ersetzt ist. Auch CARNot hat noch vor kurzer Zeit dies bei der Analyse von Krystallen aus drei Fundorten bestätigt. $^{1}$ Er fand keinen Überschufs an Kalk, doch wohl MgO und $\mathrm{FeO}$ in einem derselben (Odegarden-Norwegen), $\mathrm{FeO}$ in dem zweiten (Knappenwand, Tirol), welche Kalk ersetzt hatten. VöLCKER ${ }^{2}$

von Archaiotherium und Palaiotherium aus Nebraska (Silliman's Journ. [1854] [2] 16, 16) ergaben keinen Überschufs, für Titanotherium ein Mehr an Säure. CuAus (Journ. pr. Chem. [1852] 46, 262) analysierte einen Knochen, dessen organische Substanz ganz verschwunden war, und fand einen bedeutenden Überschufs an Basis.

\begin{tabular}{|c|c|c|c|c|c|c|}
\hline Äquivalent & Clauus & \multicolumn{3}{|c|}{ Greene } & BaUmert & IEHMANN \\
\hline Basen & $205^{3}$ & 177.4 & 184.4 & 183.2 & 194 & 197 \\
\hline Säuren · · & $179^{8}$ & 186.1 & 184.3 & 183.2 & 177 & 206 \\
\hline Differenz: & $+25^{5}$ & -8.7 & - & - & +17 & -9 \\
\hline$\%$ org. Subst. & $0 \%$ & 2.5 & 2.66 & 3.2 & $6 \%$ & $3-4 \%$ \\
\hline
\end{tabular}

Die älteren Analysen lassen die Sache also unerledigt.

1 Compt. rend. (1896) 122, 1375.

${ }^{2}$ Ber. deutsch. chem. Ges. (1883) 16, 2460. Die Krystalle waren nicht. ganz frei von fremden Substanzen (Einschlüssen), denn sie enthielten eine geringe Menge Calciumkarbonat und Sulfat, Alaunerde und unlösliche Bestand- 
hat aufserdem Apatitkrystalle von Canada und von Norwegen analysiert, in welchen ein Überschufs von Kalk enthalten ist $(1.7-5 \%$ ), jedoch derart, dals die äquivalente Menge von $\mathrm{CaF}_{2}, \mathrm{CaCl}_{2}$ und $\mathrm{CaO}$ zusammen $1 \mathrm{Mol}$. $\mathrm{CaF}_{2}$ auf $3\left(\mathrm{P}_{2} \mathrm{O}_{5} .3 \mathrm{CaO}\right)$ entspricht.

Carnot hat selbst zwei Apatite aus Canada, und einen Apatit aus den Vereinigten Staaten analysiert, in welchen die äquivalente Summe von Fluor, Chlor und Kohlensäure 1 Mol. auf 3 Mol. $\mathrm{P}_{2} \mathrm{O}_{5}$. $3(\mathrm{CaO})$ beträgt. ${ }^{1}$

Im Apatit kann also $\mathrm{F}_{2}^{\prime}$ durch $\mathrm{Cl}_{2}$, durch $\mathrm{O}$, und nach CARNoT auch durch $\mathrm{CO}_{3}$ ersetzt werden.

Diese Thatsache ist von grolsem Interesse, und erscheint annehmbar, umsomehr als schon in dem Triplordit, einem Mineral von der Krystallform und Formel des Wagnerits und Triplits F durch $\mathrm{OH}$ ersetzt gefunden ist (BRusH und DANA) ${ }^{2}$

\begin{tabular}{|c|c|c|}
\hline Wagnerit & $\mathrm{P}_{2} \mathrm{O}_{5}(\mathrm{MgO})^{8} \cdot \mathrm{MgF}_{2}$ & oder $\mathrm{PO}_{4} \mathrm{Mg} \cdot \mathrm{MgF}$ \\
\hline Triplit & 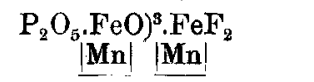 & oder $\mathrm{PO}_{4} \mathbf{F e} \cdot \mathbf{F e} \mathbf{F}$ \\
\hline Trip & $\underset{\mathrm{P}_{2} \mathrm{O}_{5} \cdot(\mathrm{MnO})^{3} \cdot \mathrm{MnOH}_{2} \mathrm{O}|| \underline{\mathrm{Fe} \mid}}{|\mathrm{Fe}|}$ & 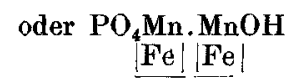 \\
\hline & $\mathrm{MnO})^{3} \cdot \mathrm{MnO} \cdot \mathrm{H}_{2} \mathrm{O}$ & ${ }_{4} \mathrm{Mn} \cdot \mathrm{MnOH}$ \\
\hline
\end{tabular}

Die Einwände, welche WEYBdLL ${ }^{3}$ gegen die Annahme VöLCKER's erhebt, kommen mir nicht entscheidend vor." Auch lehren

teile. Auch bleibt unsicher, ob das Eisenoxyd dem Phosphat oder den Einschlüssen zugerechnet werden muls. Doch bleibt feststehend, dafs für 1.7 bis $5 \% \mathrm{CaO}$ eine Säure fehlt.

1 Compt. rend. 122, 1375.

${ }^{2}$ Groth's Tabell. Utbersicht der Mineralien (Braunsehweig 1889. 3. Auf.). Ich ziehe es vor die Elemente, weIche die Bestandteile des Minerals teilweise isomorph ersetzen, unter die Formel eingeklammert zu schreiben (siehe oben S. 101). Groth und andere schreiben sie in die Formel, was die Übersicht der Formel sehr erschwert.

Ber. deutsch. chem. Ges. (1887) 20, 1525.

4 Weybull bemerkt: 1. dafs Einschlüsse in den Krystallen vorkommen köunen, und 2. dal's Apatit, besonders Chlorapatit, leicht durch das Wasser angegriffen wird und dadurch $\mathrm{CaCl}_{2}$ verloren und $\mathrm{CaCO}_{3}$ und Calciumsilikat aufgenommen haben kann. Er hält darum VöLcker's Annahme für unwahrscheinlich. Doch läfst er aufser Betrachtung, dals Säuren für den betreffenden Kalk, der 1.7-5\% beträgt, fehlen (davon ist schon abgezogen die kleine Menge $\mathrm{Kalk}$, die den gefundenen Mengen $\mathrm{CO}_{2}$ und $\mathrm{SO}_{3}$ entsprechen). Unsicher bleibt nur, ob das Eisen $(0.2-1.6 \%)$ zum Phosphat oder zu den Einschlüssen gehört. Die gefundene Alaunerde und die $\pm 1 \%$ unlöslichen Bestandteile gehören wohl zu den Einschlüssen. Ist die Formel $\left(\mathrm{Ca}_{3} \mathrm{P}_{2} \mathrm{O}_{8}\right)^{3} \cdot \mathrm{CaF}_{2}$ zu eng, sagt Werbui.L, 
die Analysen verschiedener Apatite, dals im Phosphat eine kleine Menge Kalk durch Magnesia, Manganoxydul, Eisenoxydul und Eisenoxyd ersetzt werden kann. Sie können also bis zu einer gewissen (noch unbekannten) Grenze isomorphe Bausteine in einem einzelnen Krystall bilden. ${ }^{1}$

In allen diesen Fällen entspricht jedoch die krystallinische Substanz derselben Formel: auf 3 Mol. Phosphat 1 Mol. Fluorür (resp. Chlorür, Oxyd, Hydroxyd). Jeder Baustein besteht aus einer chemischen Verbindung nach einfachem Molekularverhältnis.

Der amorphe Komplex, aus dem die kompakte Substanz der fossilen Knochen besteht, enspricht dagegen keiner chemischen Formel. Nach meiner Ansicht liegt hier noch keine Bildung einer chemischen Verbindung (des Apatits) vor, sondern (neben einer chemischen Substitution) eine Absorptionserscheinung, bei der dieselben Wirkungen stattgefunden haben, wie bei dem Komplex von Humus und kollordalem Silikat der Ackererde (und im allgemeinen bei kollordalen oder amorphen Stoffen), wenn sie mit Lösungen von Salzen, Basen, Säuren in Berührung kommen. Wie ich früher gezeigt habe, lassen sich diese Wirkungen unterscheiden in:

da könnte die Bemerkung von $\mathrm{G}_{\mathrm{RO}} \mathrm{TH}$ abhelfen, der eine Formel $\mathrm{Ca}_{5}<\begin{aligned} & \mathrm{O}_{9}\left(\mathrm{PO}_{3}\right)^{3} \\ & \left|\frac{\mathrm{Cl}}{\underline{\mathrm{OH}}}\right|\end{aligned}$

annimmt, und diese Formel verdoppelt, wenn auch Sauerstoff das Fluor vertritt. Zur Stïtze dieser Ansicht teilt WEYsuLL eine Analyse eines sehr reinen Manganapatits mit, der frei war von Wasser, Alkalien, Einschlüssen, und dazu sehr frisch. In demselben war ungefähr $1 / 10$ des Calciums durch Mangan äquivalent vertreten (Verhältnis $19 \mathrm{Ca}: 2 \mathrm{Mn}$ ). Diese Zusammensetzung palst genau auf $\mathrm{Ca}_{5} \mathrm{FO}_{9}\left(\mathrm{PO}_{3}\right)^{3}$. Diese Betrachtung scheint mir seinen $\mathrm{Zweck}$ zu verfehlen. $\mathrm{Mn}$

Denn die letzte Formel giebt dieselben prozentischen Zahlen wie $\left[\left(\mathrm{Ca}_{3} \mathrm{P}_{2} \mathrm{O}_{8}\right)^{8} \mathrm{CaF}_{2}\right]$. Mn

Nicht ein oder mehrere von diesen fünf Calciumatomen sind durch Mangan vertreten, sondern in Krystall sind Bausteine von $\left[\left(\mathrm{Mn}_{3} \mathbf{P}_{8} \mathrm{O}_{8}\right)_{8} \mathbf{M n F}_{2}\right]$ isomorpl neben den Bausteinen des Kalksalzes anwesend. Wie grofs deren Menge im Krystall sein kann, hängt von verschiedenen Umständen ab, die der Gegenstand des neu aufblühenden Studiums der Isomorphie sind. Strukturformeln können bei isomorph zusammenkrystallisierenden Verbindungen nicht aushelfen, sie sind damit unvereinbar und kommen mir daher ungereimt vor.

1 So z. B. verschiedene durch CARNor (l. c.) analysierte Apatite, einer aus Norwegen, zwei aus Canada. 
1. Chemische Substitution, nämlich Auswechselung von basischen oder von sauren Radikalen.

2. Absorption von Basen aus Salzen mit schwachen Säuren, unter Freiwerden der Säure.

3. Absorption von freien Basen, von freien Säuren, von ganzen Salzen, oder anderen Stoffen unter Bildung von AbsorptionsKomplexen.

Die dabei obwaltenden Gesetze habe ich früher untersucht und ausführlich beschrieben. ${ }^{1}$ Sie lassen sich kürzer dahin zusammenfassen, dals sich ein Gleichgewicht zwischen der Reaktion und ihrer Umkehrung einstellt, abhängig 1. von der anziehenden (absorbierenden) Kraft der absorbierenden Substanz, die selbst wieder von dem Molarbau derselben abhängt, 2 2. von der Endkonzentration (im Gleichgewichtszustande) der Lösung und der absorbierenden Substanz, 3. von der Temperatur. In der Formel $K=\frac{C_{2}}{C_{1}}$ ist also $K$ keine Konstante, sondern eine Funktion, sowohl von dem absorbierenden Stoff, wie auch von der Konzentration selber und von. der 'lemperatur.

Es kommt mir nun annehmbar vor, dafs die Änderungen im Knochen in folgender Weise auf die obengenannten Wirkungen zurückzuführen sind.

Zu 1. Die Bildung einer gewissen begrenzten Menge Manganund Ferriphosphat durch eine begrenzte Substitution von Ca durch $\mathrm{Mn}$ und $\mathrm{Fe}^{\prime \prime \prime}$. Ebenso die Bildung von $\mathrm{CaF}_{2}$ durch eine begrenzte Substitution von $\mathrm{CO}_{3}$ des $\mathrm{CaCO}_{3}$ im Knochen durch Fluor.

Zu 2. Die Absorption von $\mathrm{CaO}$ aus dem Bikarbonat des Bodenwassers durch das Phosphat. Diese Aufnahme ist analog der Aufnahme von Alkali, die ich bei der kolloidalen $\mathrm{SiO}_{2}$ (auch der bei $15^{0}$ entwässerten) aus einer Lösung eines Alkalikarbonats be-

1 Journ. pr. Chem. (1881) 23, 324-349 und 379-495, Landw. Versuchsstationen (1888) 35, 69-136, Zeitschr. phys. Chem. (1895) 1s, 331.

$\approx$ So hat 1. das Kolloïd von $\mathrm{SiO}_{2}$ (der Hydrogel der Kieselsäure) ein kleineres Absorptionsvermögen für Säuren und auch für Salze als das Kolloüd von $\mathrm{SnO}_{2}$, 2. ist das Absorptionsvermögen von $\mathrm{SnO}_{2}$ verschieden, je nachdem der Hydrogel durch die Zeit, die Temperatur und andere Ursachen in seinem Bau modifiziert ist (Journ. pr. Chem. [1881] 23, 337-338).

${ }^{3} C_{1}$ ist die Konzentration der Lösung, $C_{2}$ dic Konzentration (=Gehalt) des absorbierenden Körpers an der (aus der Lösung) absorbierten Substanz).

z. azorg. Chem. XV. 
obachtete. ${ }^{1}$ Dabei wird Alkalibikarbonat gebildet. Wie stark solche Wirkungen sein können, beweist das rote Kollord von $\mathrm{MnO}_{2}{ }^{2}$, das aus einer Lösung von $\mathrm{K}_{2} \mathrm{SO}_{4}$ (auch $\mathrm{KNO}_{3}, \mathrm{KCl}$ ) nicht allein $\mathrm{K}_{2} \mathrm{SO}_{4}$, sondern auch Alkali absorbiert und Schwefelsäure (resp. Salpetersäure, Salzsüure) frei macht. ${ }^{2}$

Von dem amorphen Phosphat des Knochens darf man eine Absorption annehmen; umsomehr als schon BLAREz gefunden hat, dafs Phosphorsäure mit überschüssigem Kalkwasser versetzt einen kolloqdalen Niederschlag bildet, der mehr als 3 Mol. CaO entbält. Er fand bei seinen Versuchsbedingungen einen Gehalt von 3.6 Mol., welcher selbst nach dem Auswaschen noch 3.3 Mol. betrug. Im Baryumsalze erhielt er unter denselben Umständen 3.45 Mol. BaO. ${ }^{3}$ Es leuchtet ein, dals diese Mengen nur zufällige sein können, weil sie abhängen von den Enakonzentrationen der Lösung und des Kolloirds, die bei der Temperatur des Versuches und bei den angewandten Mengen der Lösungen den Gleichgewichtszustand bildeten.

$\mathrm{Zu}$ 3. Annehmbar, wenigstens sehr wahrscheinlich, ist die Anziehung des Knochenphosphats auf die im Bodenwasser gelösten Phosphate und kohlensauren Kalke und auf das sich bildende Fluorcalcium, derart, dals sich ein Absorptionskomplex bildet. An diesem Komplex nehmen dann auch wahrscheinlich das Mangan- und Eisenphosphat teil. Bezeichnend dafür ist, dafs das Phosphat, nachdem schon $4 / 5$ desselben von Essigsäure gelöst war, noch ungefähr dieselbe basische Zusammensetzung besals als vorher (siehe S. 102).

Denn die Absorption ist für kolloüdale und amorphe Stoffe eine gewöhnliche Erscheinung. Sie kann sehr stark sein. Der kollordale Komplex von Humus and leicht zersetzbaren Silikaten in der Ackererde - Kollorde wie die Hydrogels (oder andere Gels) ron $\mathrm{SiO}_{2}$, $\mathrm{SnO}_{2}, \mathrm{Al}_{2} \mathrm{O}_{3}, \mathrm{Cr}_{2} \mathrm{O}_{3}, \mathrm{MnO}_{2}$ - die kollordalen Niederschläge ron Phosphaten, Karbonaten, Boraten - amorphe Niederschläge wie von $\mathrm{BaSO}_{4}$ - alle diese Körper absorbieren in ihrem Gewebe allerlei Stoffe aus wässeriger Lösung mit einer Kraft, die mit der Zunahme der absorbierten Menge abnimmt. Für kleine Mengen kann die Bindung äufserst stark sein. Darf man eine solche Anziehung im fossilen Knochen annehmen, so mufs sie die Ausfüllung

1 Nach Frésy bereitet aus $\mathrm{KMnO}_{4}$ und $\mathrm{H}_{2} \mathrm{SO}_{4}$.

2 Journ. pr. Chem. (1881) 23, 344. Siehe weiter die isothermische Kurve $\left(C_{1}, C_{2}\right)$ für diese Absorption von $\mathrm{K}_{2} \mathrm{SO}_{4}$ in Zeitschr. phys. Chem. (1895) 18, 333.

- Compt. rend. (1887) 104, 270. 
der durch das Verschwinden des Osserns in den Lamellen entstandene Leere mit $\mathrm{CaF}_{2}, \mathrm{CaO}, \mathrm{H}_{2} \mathrm{O}$ und $\mathrm{CaCO}_{3}$ befördern. ${ }^{1}$ Was solche Absorptionskomplexe betrifft, so ist es wahrscheinlich, dafs schon der frische Knochen ein solches darstellt, also von $\mathrm{Ca}_{3} \mathrm{P}_{2} \mathrm{O}_{8}$ mit wenig $\mathrm{Mg}_{3} \mathrm{P}_{2} \mathrm{O}_{8}$ und viel $\mathrm{CaCO}_{3}$ - nach GABRIEL auch mit $\mathrm{Ca}(\mathrm{OH})_{2}$. Eine chemische Formel (und noch viel weniger eine Strukturformel) darauf anzuwenden, wie GABRIEL für frische Knochen und AEBY für frische und fossile Knochen thut, hat nach meiner Ansicht keinen Sinn. Es liegt hier keine chemische Verbindung, sondern ein Absorptionskomplex vor.

Die Absorptionswirkungen in den fossilen Knochen müssen dem Gesetze gehorchen, dafs ein Gleichgewicht sich einstellt zwischen dem absorbierenden Körper und den im Bodenwasser gelösten Bestandteilen. Aufserdem kann der absorbierende Körper Modifikationen erleiden, wodurch der Verteilungsfaktor sich ändert und also die Absorption vermehrt oder verringert wird.

Es entsteht daher die Frage: wie weit ist die Absorption im Phosphat (des Stegodonknochens) fortgeschritten. Ist dasselbe mit dem Bodenwasser der Schicht, worin der Knochen eingebettet war, ins Gleichgewicht gekommen, und also mit den absorbierten und substituierten Bestandteilen dem Bodenwasser gegenüber schon

1 Wird es bestätigt, dafs neues Calciumphosphat aufgenommen wird, daun ist dies vielleicht sehr geeignet, solche Anziehungen auszuüben.

2 GABRIEL nimmt an $\left(\mathrm{Ca}_{3} \mathrm{P}_{2} \mathrm{O}_{8}\right)^{5} \cdot \mathrm{Ca}(\mathrm{OH})^{2} \cdot 2 \mathrm{H}_{2} \mathrm{O}$. Fin kleiner Teil des $\mathrm{CaO}$ ist durch $\mathrm{MgO}, \mathrm{Na}_{2} \mathrm{O}, \mathrm{K}_{2} \mathrm{O}$ ersetzt. Aesy macht die Hypothese, dals das $\mathrm{CaCO}_{3}$ in zwei Zuständen vorkommt; ein Teil wäre mit dem $\mathrm{Ca}_{3} \mathrm{P}_{2} \mathrm{O}_{8}$ apatitartig verbunden, ein Teil nicht. Also enthält der frische Knochen naeh Assr: $\left(\mathrm{Ca}_{3} \mathrm{P}_{2} \mathrm{O}_{8}\right)^{3} \cdot \mathrm{CaCO}_{3}$ nebst einer Menge von freiem $\mathrm{CaCO}_{3}$ und $\mathrm{MgCO}_{3}$. Im $\mathrm{Zahm}$ schmelz nimmt er nur $\mathrm{Ca}_{3} \mathrm{P}_{2} \mathrm{O}_{8}$ nebst freiem $\mathrm{CaCO}_{3}$ an. Die Kohlensäure in Phosphat sollte schon unter Rotglühhitze ausgetrieben und durch Behandlung mit Ammoniumkarbonat nicht wieder anfgenommen werden. In den fossilen Knochen nimmt er die Formel $\left[\mathrm{Ca}_{3} \mathrm{P}_{2} \mathrm{O}_{8}\right]^{3} \mathrm{CaCO}_{3} \cdot \mathrm{H}_{3} \mathrm{O}$ an. Nur der $\mathrm{CaCO}_{3}$, $(\mathrm{OH})_{2}$

der an Phosphat gebunden ist, kann durch Einwirkung mit alkalischen Fluorüren in $\mathrm{CaF}_{2}$ umgesetzt werden. Dagegen setzt der $\mathrm{Ca}_{3} \mathrm{P}_{2} \mathrm{O}_{8}$ des Zahnschmelzes sich in Vivianit um. Das $\mathrm{MgCO}_{3}$ setzt sich in Karbonate von $\mathrm{Mn}, \mathrm{Fe}, \mathrm{Ca}$ um. Aeby stiutzt diese Hypothesen 1. auf seine Analysen der frischen und der fossilen Knochen aus den Pfahlbauten, 2. darauf, dals die Knochen (wie such das Zahnbein) eine andere Umsetzung erlitten haben als der Zahnschmelz. Seine Ansichten bervhen wohl auf zu schwachen Gründen, um annehmbar zu sein. Das Verhalten der Kohlensänre ist durch WIBen (Ber. deutsch. chem. Ges. [1874] 7, 555) widerlegt. Doch sind AEBy's Beobachtungen immerhin bemerkenswert. 
gesättigt gewesen? Man muls dabei in Betracht ziehen, dals die absorbierende Substanz $(A)$ aus dem Wasser gelöste Stoffe $(a, b, c$ u. s. w.) absorbiert, bis die erhaltene Konzentration von $A$ an den Stoffen $a, b, c$ mit der restierenden Konzentration des Wassers ein Gleichgewicht bildet. Wird dann das Bodenwasser ernevert, dann kann $A$ eine neue Menge von $a, b, c \ldots$ absorbieren. Die absorbierte Menge nimmt bei jeder Erneuerung ab, und schliefslich mufs eine Konzentration von $A$ erhalten werden, die nicht weiter wächst. Vom Fluorcalcium kann man annehmen, dals es sehr lange Zeit dauert, bis diese Endkonzentration eintritt, 1. weil der Gehalt des Bodenwassers an Fluor so verschwindend klein ist, 2. weil die absorbierende Kraft des Phosphates für $\mathrm{CaF}_{2}$ verhältnismäfsig grofs ist und dasselbe also noch einer höchst verdünnten Lösung des Fluors das Gleichgewicht halten kann, wenn es schon bis zu $1 \mathrm{Mol}$. $\mathrm{CaF}_{2}$ absorbiert hat. Von den übrigen absorbierten Stoffen $\mathrm{CaO}, \mathrm{MnO}, \mathrm{Fe}_{2} \mathrm{O}_{3}$ lälst sich in dieser Hinsicht noch nichts Sicheres behaupten. Unsere Kenntnisse über die Absorption sind noch zu dürftig, namentlich weil hier der Zeitfaktor eine bedeutende Rolle spielen muls und Modifikationen in der absorbierenden Sub$\operatorname{stanz}$ hervorrufen kann, die uns noch ganz unbekannt sind.

Die amorphen und kolloïdalen Absorptionsverbindungen können unter rerschiedenen Umständen wie: Zusammensetzung und Konzentration der Lösung, Druck, Temperatur, Modifikation u. s. w., mit verschiedener Geschwindigkeit in chemische Verbindungen übergehen, die einem einfachen Atom- oder Molekularverhältnisse entsprechen. Sie sind in vielen Fällen die Vorläufer von krystalloïdalen wahren chemischen Verbindungen, wovon ich früher Beispiele gegeben habe. ${ }^{1}$ Das ist bei Hydraten, Phosphaten, Sulfüren u. s. w. oft beobachtet. So kann sich auch ein krystallinisches Calciumphosphat $\mathrm{P}_{2} \mathrm{O}_{5} .4 \mathrm{CaO}$ bilden. ${ }^{2}$ Die Annahme also, dals in den fossilen Knochen keine Bildung von Apatit, sondern eine Absorption von $\mathrm{CaF}_{2}, \mathrm{CaO}$ u. s. w. stattgefunden hat, schliefst nicht aus, ja kann die notwendige $\mathrm{Be}$ dingung sein, dals sich in der Natur unter geeigneten Umständen aus einem solchen Absorptionskomplex, wie ihn der fossile Knochen jetzt darstellt, ein krystallinischer Apatit bilden kann. Dabei können verschiedene Phosphate und Fluorüre, die isomorph zusammen

1 Hydrat von $\mathrm{BeO}$ und von MgO aus dem Hydrogel derselben (J.pr. Ch. [1882] 26, 227). Hydrat von $\mathrm{Al}_{2} \mathrm{O}_{3}$ (Rec. d. Tr. Ch. Paysbas [1888] 7, 82-87).

"In den Thomasschlacken gefunden. 
zu krystallisieren vermögen, Bausteine desselben Krystalles werden. Denn die Natur liefert Apatite, die eine gewisse Menge $\mathrm{MnO}, \mathrm{MgO}$, $\mathrm{FeO}, \mathrm{Fe}_{2} \mathrm{O}_{3}$ enthalten, welche den Kalk äquivalentgemäls ersetzen, wie oben besprochen ist.

\section{Schlüsse.}

Im fossilen Knochen des Stegodons aus der Tertiärzeit, in einer Lapillischicht unter einem zum Sandstein erhärteten vulkanischen Tuff, wurde das Folgende gefunden:

Die Struktur ist erhalten, die HaFER'schen Kanäle und der spongiöse Teil ist mit krystallinischem $\mathrm{CaCO}_{3}$ ausgefüllt. In der äufseren Schicht des kompakten Teiles und im spongiösen Teil ist Pyrit abgesetzt. Die organischen Teile des Gewebes sind grölstenteils verschwunden.

Aus der Erhaltung der Struktur, aus dem erhöhten spez. Gewicht und ans der Analyse lärst sich ableiten, dals aus dem Bodenwasser nicht allein $\mathrm{CaCO}_{3}$, sondern auch Calciumphosphat im Gewebe des kompakten Teiles aufgenommen ist. Ein Teil des Calciumkarbonats ist in Fluorcalcium $(3 \%)$ metamorphosiert; desgleichen die Hälfte des Magnesiumphosphates und ein kleiner Teil des Calciumphosphates in Mangan- und Ferriphosphat. Aufserdem ist das Phosphat durch Aufnahme von Kalk basisch geworden, sodals die Basen mit ungefähr $1 / 8$ ihres Betrages die Säuren übertreffen, was auf $2 \mathrm{Mol}$. Orthophosphat fast $1 \mathrm{Mol}$. Basis ausmacht.

Es ist anzunehmen, dals diese Aufnahme von Phosphaten, ron $\mathrm{CaF}_{2}$, von $\mathrm{CaCO}_{3}$, von $\mathrm{CaO}$, eine Folge der Absorptionsanziehung des ursprünglichen Phosphates ist. Das Ganze bildet keine chemische Verbindung, sondern einen wasserhaltigen Absorptionskomplex.

Es ist wahrscheinlich, dafs daraus unter geeigneten Umständen ein krystallinischer Apatit als chemische Verbindung entstehen kann, und dafs dieser Apatit verschiedene Phosphate (mit $\mathrm{CaF}_{2}$ und mit $\mathrm{CaO}$ ) als isomorphe Bausteine enthalten kann.

Neue Untersuchungen von frischen Knochen sind erwünscht in Betreff 1. des Wassergehaltes, 2. der Frage, ob das Orthophosphat darin schon in geringem Mafse basisch ist.

\section{Anhang.}

Die Bestimmungsmethoden.

Die Bestimmung der organischen Substanz, des Schwefels und des Wassers im Knochen bietet Schwierigkeiten, indem bei der Einäscherung an der Luft: 
1. die Kohlensäure teilweise ausgetrieben,

2. das anwesende $\mathrm{FeS}_{2}$ in $\mathrm{Fe}_{2} \mathrm{O}_{3}$ umgesetzt wird, und es die Frage ist, ob der Schwefel zu $\mathrm{SO}_{2}$ oder $\mathrm{SO}_{3}$ oxydiert wird, und $o b$ er teilweise entweicht oder ganz durch den kohlensauren Kalk zurückgehalten wird,

3. das Manganoxydul oxydiert wird, wodurch die Asche eine grüne Farbe annimmt und mit Salzsäure eine kleine Menge Chlor entwickelt.

4. die organische Substanz und das über $110^{\circ}$ gebundene Wasser aus der Elementaranalyse und dem Glühverlust nur annähernd bestimmbar sind.

Die Menge des Glühverlustes mufs also manche Korrekturen erhalten, was früher bei Analysen von dergleichen Substanzen wohl vielfach übersehen ist.

Ich befolgte dieselbe Methode, die ich früher bei Ackererden angewandt und ausführlich beschrieben habe. ${ }^{1}$

Schwefelbestimmung. Es hat sich herausgestellt, 1. dafs der Pyrit nach Ausziehung mit Salzsäure zurückblieb und 2. dafs bei der Erhitzung der Substanz im Luftstrome der Schwefel aus dem Pyrit zu $\mathrm{SO}_{3}$ verbrannte und durch den Kalk ganz oder fast ganz zurückgehalten wurde (siehe Spalte 2, 4, 5). Der Schwefelgehalt ist aus der erhaltenen Schwefelsäure berechnet:

Substanz B.

\begin{tabular}{l|c|c|c|c}
\hline & $\begin{array}{c}\text { In der Sub- } \\
\text { stanz mit Soda } \\
\text { und Salpeter } \\
\text { geschmolzen }\end{array}$ & $\begin{array}{c}\text { Im } \\
\text { wässerigen } \\
\text { Auszug }\end{array}$ & $\begin{array}{c}\text { Im Rückstand } \\
\text { des } \\
\text { salzsauren } \\
\text { Auszugs }\end{array}$ & $\begin{array}{c}\text { In } \\
\text { der Substanz } \\
\text { nach Glühen } \\
\text { im } \\
\text { Luftstrome }\end{array}$ \\
\hline $\begin{array}{l}\text { Schwefel . . . } \\
\text { Eisen. . . . . }\end{array}$ & $1.36 \%$ & $0.00 \%$ & $1.36=4.25$ Atome & $1.30 \%, 1.26 \%$ \\
\hline $\begin{array}{c}\text { Verhältnis d. Atome } \\
\text { S zu Atomen Fej }\end{array}$
\end{tabular}

Der Schwefel war also nur als Pyrit im unlöslichen Teil anwesend.

1 Über die Bestimmung des Wassers, des Humus, des Schwefels und der in kollö̈dalen Silikaten gebundenen Kieselsäure (Landw. Versuchsstationen [1890] 37, 279). 
Die organische Substanz. Sie wurde abgeleitet aus der bei der Elementaranalyse enthaltenen Kohlensäure und dem Wasser, und aus der Kohlensäurebestimmung 1. in der Substanz, 2. in dem Rückstand der Elementaranalyse. Der daraus berechnete organische Kohlenstoff wurde multipliziert mit dem Faktor von Wouff (1.724). In dieser organischen Substanz wurde der Wasserstoffgehalt zu 4\% angenommen. Obgleich diese Bestimmungen die Menge organischer Substanz nur annähernd ergeben, so kann der Fehler doch nur einzelne Zehntel Prozent betragen.

So wurde gefunden:

$\begin{array}{llllllllll}\text { In } A_{1} & . & . & . & . & . & . & 2.24 \% & \text { organische Substanz. } \\ \text { In } B & . & . & . & . & . & . & 1.7 & & \\ \text { In } A_{2} & . & . & . & . & . & . & 1.58 & & \end{array}$

Wasser. Dieses mulste aus den Zahlen des Glühverlustes und der Elementaranalyse annähernd bestimmt werden.

Es ergaben sich dabei Differenzen, die bei einer näheren Untersuchung sich als die Folge davon erwiesen, dals im Rohr der Elementaranalyse und selbst im Muffelofen der Glühverlust um so höher war, je stärker die Hitze gewesen war. Bei Erhitzung über der Gebläseflamme wurde ein neuer Glühverlust beobachtet.

Da GABRIEL bei seinen Knochenanalysen noch $\pm 1 \%$ Gewichtsverlust beobachtet hat, wenn er den schon über der Gebläseflamme bis zur Gewichtskonstanz erhitzten Stoff mit $\mathrm{SiO}_{2}$ mischte und dann diese Erhitzung wiederholte, ${ }^{1}$ haben wir dasselbe gethan. Wir erhielten dabei noch $\pm 0.5 \%$ Verlust.

Der beobachtete Glühverlust - entweder im Muffelofen oder im Rohr der Elementaranalyse - wurde korrigiert: für die ausgetriebene Kohlensäure, indem die Kohlensäure in der ursprünglichen Substanz und in dem Glühverlust bestimmt wurde, für den aufgenommenen Sauerstoff aus dem Pyritgehalt, aus der Schwefelsäurebestimmung im Glührückstand und aus dem Mangangehalt. Die qualitative Analyse hatte erwiesen, dals das Eisen, welches nicht als Pyrit vorkam, schon in der ursprünglichen Knochensubstanz als $\mathrm{Fe}_{2} \mathrm{O}_{3}$ anwesend war.

1 Gabriel macht darauf aufmerksam, dafs bei den allermeisten Knochenanalysen ein Defizit übrig bleibt, das die Untersucher durch Annahme von $\mathrm{CaF}_{2}$ gedeckt haben. Jedoch die normale Menge $\mathrm{CaF}_{2}$ ist nur höchst gering, kleiner wie $0.1 \%$. Auch bei seinen mit der höchsten Genauigkeit ansgeführten Analysen kommt ein Defizit von $1 \%$, das jedoch hinwegfallt, wenn er diesen erst durch $\mathrm{SiO}_{2}$ bewirkten Glühverlust in Rechnung bringt. 
Die Menge stärker gebundenen Wassers wurde bei den Elementaranalysen als durch Chlorcalcium aufgenommenes Wasser gefunden wie folgt:

\begin{tabular}{l|c|c|c}
\hline \hline & $A_{1}$ & $C$ & $B$ \\
\hline \hline $\begin{array}{r}\text { Nach Abzug des Wasserstoffes in } \\
\text { der organischen Substanz . . . }\end{array}$ & $5.48 \%$ & $6.49 \%$ & $5.8 \%$ \\
Bei $110^{\circ}$ ausgetrieben . . . . & $4.17 \%$ & $4.4 \%$ & $3.7 \%$ \\
\hline Also stark gebundenes Wasser. . & $1.35 \%$ & $\mathbf{2 . 0 9 \%}$ & $2.1 \%$
\end{tabular}

Die dabei angewandte Glühhitze namentlich bei $A_{1}$ war nicht stark. Beim Erhitzen im Muffelofen während verschiedener Stunden wurde ein grölserer Verlust berechnet. Um zu untersuchen, ob das Fluorcalcium dabei unverändert blieb, wurde eine Schale mit reinem $\mathrm{CaF}_{2}$ daneben gestellt; das Gewicht blieb konstant. Es wurde also kein Fluor durch Sauerstoff ersetzt. Die Erhitzung über der Gebläseflamme bis zum konstanten Gewicht, welches nach 10 Minuten erhalten wurde, gab einen neuen Verlust. Dieser Verlust vermehrte sich in zwei Versuchen noch um $0.5-0.6 \%$, nachdem der Stoff mit $\mathrm{SiO}_{2}$ gemischt war.

Schliefslich wurden also aus dem Glühverlust die folgenden Mengen Wasser berechnet:

\begin{tabular}{|c|c|c|c|}
\hline & \begin{tabular}{|c|}
$B$ \\
Glühen \\
in der Muffel
\end{tabular} & $\begin{array}{c}B \\
\text { Glühen } \\
\text { in der Muffel }\end{array}$ & $\begin{array}{c}B_{1} \\
\text { Glïhen in der-Muffel, } \\
\text { nachher im Gebläse } \\
\text { und mit } \mathrm{SiO}_{2}\end{array}$ \\
\hline Korrigierter Glühverlust . & $8.45 \%$ & $8.51 \%$ & $10.54 \%$ \\
\hline $\begin{array}{l}\text { Organische Substanz }+ \text { bei } \\
110^{\circ} \text { ausgetriebenes Wasser }\end{array}$ & 5.39, & 5.39, & 6.05 \\
\hline Also stark gebundenes Wasser & $3.06 \%$ & $3.12 \%$ & $4.49 \%$ \\
\hline
\end{tabular}

$A$ ergab bei längerem Glühen $4.5 \%$ und $C$ auf dieselbe Weise wie $B_{1}$ behandelt, $3.3 \%$.

Die Menge des Wassers, welche bei $110^{\circ}$ noch nicht ausgetrieben wird, ist also auf 3.5-4.5 zu stellen, wovon jedoch ein Teil erst bei sehr starker Glühhitze entweicht.

Die Analysen von $A$ und $B$ (siehe S. 95) verlangen 4.4 und $4.2 \%$, um auf $100 \%$ zu schlielsen. 
Mineralische Bestandteile. Die bei mälsiger Rotglühhitze im Muffelofen von dem organischen Teil befreite Substanz wurde in einer Platinschale mit Salpetersäure wiederholt eingeengt und von einer geringen Menge unlöslichen Rückstandes abfiltriert. Im Filtrat wurden $\mathrm{P}_{2} \mathrm{O}_{5}$ u. s. w. bestimmt.

Phosphorsäure. Diese wurde nach zwei Methoden von den übrigen Bestandteilen getrennt, 1. nach CHANCEL mit Wismutnitrat in der salpetersauren Lösung der Asche und 2. nach Отто (als basisches Eisenphosphat) in der salzsauren Lösung der Asche. Die erste Methode hatte den Vorteil, dafs das Filtrat die Basen als Nitrate enthielt und Eisen und Mangan (nach Entfernung des Wismuts) nach der Methode von St. Clatre Deviture von Kalk, Magnesia und Alkalien durch Wasser getrennt wurden. Diese Methode ist bequem und genau. Nur muls man sich überzeugen, ob die Phosphorsäure vollständig durch das Wismutnitrat abgeschieden ist. In $A$ (siehe die Tabelle auf Seite 95 unten) wurde das eine Mal noch eine sehr geringe Menge $(0.06 \%)$, das andere Mal nur eine Spur Phosphorsäure beim Eisen und Mangan aufgefunden. Die zwei $\mathrm{P}_{2} \mathrm{O}_{5}$-Bestimmungen stimmen ganz befriedigend unter einander und mit der dritten, bei der das $\mathrm{P}_{2} \mathrm{O}_{5}$ als basisches Eisenphosphat abgeschieden wurde. Bei der Einengung der salpetersauren Lösung der Asche zeigte sich die Erscheinung, dals ein in Salpetersäure unlösliches Salz sich absetzte, das Phosphorsäure und Manganoxyd enthielt. ${ }^{1}$ Es war also nötig, die Einengung nicht soweit zu treiben, dals die Absetzung dieses Salzes anfing.

Mangan und Eisen. Das nach der Methode Devilute erhaltene und gewogene Gemisch von $\mathrm{Fe}_{2} \mathrm{O}_{3}$ und $\mathrm{MnO}_{2}$ (oder $\mathrm{Mn}_{3} \mathrm{O}_{4}$ ) wurde ins Nitrat verwandelt und dann in der einen Hälfte das Eisen nach der Methode Oudemans bestimmt, in der anderen Hälfte das Mangan nach der Methode $\mathrm{H}_{\mathrm{AMPE}}{ }^{2}$ mit $\mathrm{KClO}_{3}$. Auch wurden Mangan und Eisen durch Ammoniak getrennt. Beide Methoden lieferten übereinstimmende Zahlen.

Kalk, Magnesia. Der Kalk wurde zweimal als Oxalat gefällt, um die Trennung von der Magnesia vollständig zu machen. Das

${ }_{1}$ In Salpetersäure unlösliches Manganiphosphat $\mathrm{Mn}_{2} \mathrm{O}_{3} \mathrm{P}_{2} \mathrm{O}_{5}$ ist beschrieben von O. Christensen (Journ. pr. Ohem. [1883] 28, 20). Grünlichgraues Pulver. Wurde die Asche des fossilen Knochens in verdünnter Salpetersäure gelöst, so kam das Manganiphosphat mit blauer Farbe zum Vorschein.

${ }^{2}$ Chem. Ztg. 1885, 1083. 
Filtrat wurde zur Trockne eingedampft, die Ammoniaksalze ausgetrieben, und die Magnesia im Rückstand bestimmt.

Kohlensäure. Die Kohlensäure wurde unter allen nötigen Vorsichtsmafsregeln in einem geeigneten Apparat ausgetrieben und in Natronkalk aufgenommen. Dafs auf diese Weise genaue Zahlen erhalten wurden, bewiesen zwei Kontrollanalysen:

$$
\begin{array}{lcc} 
& \text { Berechnet: } & \text { Gefunden: } \\
\mathrm{Na}_{2} \mathrm{CO}_{3} & 205.2 \mathrm{mg} & 205.2 \mathrm{mg} \\
\mathrm{CaCO}_{3} & 222.3 " & 221.9 "
\end{array}
$$

Das Kali wurde sowohl in einem Auszug mit siedendheifsem Wasser, wie in der Lösung, die nach DeviLue's Methode von den übrigen Basen getrennt war, als Platindoppelsalz bestimmt.

Schliefslich bezeige ich den Herren Klobbie und Simon Thomas meinen freundlichen Dank für ihre wertvolle Hilfe bei der Ausführung der Analysen.

Leiden, Anorg. chem. Universitäts-Laboratorium, Mai 1897.

Bei der Redaktion eingegangen am 29. Mai 1897. 